\title{
Photosynthetic Responses of Greenhouse Ornamentals to Interaction of Irradiance, Carbon Dioxide Concentration, and Temperature
}

\author{
Ian K. Atkins \\ Department of Biological Systems Engineering, University of Wisconsin-Madison, 460 Henry \\ Mall, Madison, WI 53706
}

\author{
Jennifer K. Boldt \\ U.S. Department of Agriculture, Agricultural Research Service, Application Technology Research \\ Unit, 2801 W. Bancroft Street, Mail Stop 604, Toledo, OH 43606
}

\begin{abstract}
Additional INDEX wORDs. calibrachoa, carbon dioxide enrichment, environmental control, floriculture, geranium, pepper, petunia, sunflower, verbena

Abstract. Supplemental lighting, temperature control, and $\mathrm{CO}_{2}$ enrichment can improve the productivity of greenhouse crops, but operating costs for greenhouse control systems to maintain environmental parameters at desired setpoints can be expensive. To balance operating costs with productivity, growers need to be able to predict how a crop will perform as a function of photosynthetic photon flux density (PPFD), $\mathrm{CO}_{2}$ concentration, and temperature. The objective of this study was to explore the response of net photosynthetic rate $\left(\mathrm{P}_{n}\right)$ to $P P F D$ and $\mathrm{CO}_{2}$ concentration, for plants acclimated to different growth environment temperatures or light intensities. We measured $P_{n}$ at all combinations of 14 irradiances and four $\mathrm{CO}_{2}$ concentrations of calibrachoa (Calibrachoa $\times$ hybrida 'Superbells Lemon Slice'), petunia (Petunia $\times$ hybrida 'Supertunia Mini Strawberry Pink Veined'), and verbena (Verbena $\times$ hybrida 'Superbena Royale Whitecap') grown at three light intensities, and of geranium (Pelargonium $\times$ hortorum 'Maverick Red'), pepper (Capsicum annuum 'California Wonder'), and sunflower (Helianthus annuus 'Pacino Gold') grown at three different temperatures. Sunflower, pepper, and geranium were fit to a model representing $P_{n}$ as a function of $P P F D, \mathrm{CO}_{2}$ concentration, and leaf temperature. Photosynthetic light response curves, at each $\mathrm{CO}_{2}$ concentration, were fit for each species and growth environment using a nonrectangular hyperbola. These models can be used to identify multiple combinations of $P P F D, \mathrm{CO}_{2}$ concentration, and leaf temperature that would result in equivalent rates of photosynthesis, allowing the most cost-effective combination to be chosen.
\end{abstract}

Many greenhouse-grown plants are started in late winter or early spring when outdoor light intensity and air temperature are lower than ideal for plant growth (Korczynski et al., 2002). To reduce heating costs, greenhouses are typically sealed as tightly as possible, which can contribute to low $\mathrm{CO}_{2}$ concentrations inside (Mortensen, 1987). Conversely, greenhouses often require some form of cooling during summer months, typically through use of vents, fans, or evaporative cooling. The timing of $\mathrm{CO}_{2}$ enrichment should be considered, as the increased air exchange rate during periods of active ventilation, either for temperature or humidity control, minimizes its efficacy. Supplemental lighting, temperature control, and $\mathrm{CO}_{2}$ enrichment can improve the productivity of greenhouse crops, but capital costs for climate

Received for publication 28 July 2021. Accepted for publication 15 Nov. 2021. Published online 21 January, 2022.

Mention of trade names or commercial products in this publication is solely for the purpose of providing specific information and does not imply recommendation or endorsement by the U.S. Department of Agriculture (USDA). USDA is an equal opportunity provider and employer.

This study was supported by the National Science Foundation Graduate Research Fellowship Program (Grant No. DGE-1256259) and an internship provided through the Graduate Research Internship Program. Any findings and conclusions or recommendations expressed in this material are those of the author(s) and do not necessarily reflect the views of the National Science Foundation.

J.K.B. is the corresponding author. E-mail: jennifer.boldt@usda.gov.

This is an open access article distributed under the CC BY-NC-ND license (https://creativecommons.org/licenses/by-nc-nd/4.0/). control systems and operating costs for these systems to control heaters, lights, vents, fans, and/or supplemental $\mathrm{CO}_{2}$ systems to maintain environmental parameters at desired setpoints can be expensive. To balance operating costs with productivity, growers need to be able to predict how a crop will perform as a function of PPFD, $\mathrm{CO}_{2}$ concentration, and temperature. Determining which combinations can provide similar net photosynthetic rates $\left(\mathrm{P}_{\mathrm{n}}\right)$ can assist growers in evaluating which combination is most cost-effective for their operation. Net photosynthetic rate is a useful proxy for a plant's instantaneous productivity and is about proportional to dry matter accumulation (Seginer et al., 1991).

Plant photosynthetic response to $P P F D$, or photosynthetically active radiation $(P A R)$ intensity, has been characterized in many studies, both empirically (e.g., Acock, 1991; Johnson and Thornley, 1984; Pachepsky and Acock, 1996) and biochemically (see review by von Caemmerer et al., 2009). Additional studies have examined photosynthetic response to $\mathrm{CO}_{2}$ concentration and temperature within the context of increasing atmospheric $\mathrm{CO}_{2}$ concentration and climate change (e.g., Idso et al., 1987), although much of this research has focused on field crops (Bunce and Heichel, 1986). Research focused on measuring photosynthetic responses of greenhouse crops has typically varied only one factor (irradiance, temperature, or $\mathrm{CO}_{2}$ concentration) at a time. Boldt et al. (2014) measured leaf photosynthetic response, singly, to irradiance, $\mathrm{CO}_{2}$ concentration, and leaf temperature of 13 herbaceous ornamentals, and the models were incorporated into a decision-support tool for floriculture growers, 
PhotoSim [U.S. Department of Agriculture, Agricultural Research Service (USDA-ARS), 2019]. Erwin and Gesick (2017) measured leaf photosynthetic response of 10 taxa of leafy greens grown in greenhouses to PPFD and $\mathrm{CO}_{2}$ concentration. In both Boldt et al. (2014) and Erwin and Gesick (2017), $\mathrm{CO}_{2}$ concentration was maintained at $400 \mu \mathrm{mol} \cdot \mathrm{mol}^{-1}$ for the light response curves, and $P P F D$ was maintained at $300 \mu \mathrm{mol} \cdot \mathrm{m}^{-2} \cdot \mathrm{s}^{-1}$ for the $\mathrm{CO}_{2}$ response curves. Therefore, data gathered in these studies allowed for modeling of $\mathrm{P}_{\mathrm{n}}$ along two intersecting lines in a domain of the variables $\mathrm{CO}_{2}$ concentration and $P P F D$, but not in the rest of the plane. Stanghellini and Bunce (1993) measured leaf $\mathrm{P}_{\mathrm{n}}$ of tomato (Solanum lycopersicum) at all combinations of $\mathrm{CO}_{2}$ concentration (100 to $1000 \mu \mathrm{mol} \cdot \mathrm{mol}^{-1}$ ), PPFD (50 to $2000 \mu \mathrm{mol} \cdot \mathrm{m}^{-2} \cdot \mathrm{s}^{-1}$ ), and temperature $\left(18,25\right.$, and $\left.32^{\circ} \mathrm{C}\right)$, providing insight into the response of $\mathrm{P}_{\mathrm{n}}$ over the $P P F D$ and $\mathrm{CO}_{2}$ concentration domain, as well as their interaction with temperature. This allows $\mathrm{P}_{\mathrm{n}}$ to be estimated via interpolation at any combination of $\mathrm{CO}_{2}$ concentration, temperature, and PPFD within the domain tested, which encompasses the range of parameters likely to occur for tomato production. Because it is known [e.g., results from Boldt et al. (2014) and Erwin and Gesick (2017)] that photosynthetic responses vary significantly between species, similar data for additional plant species are warranted.

It would be useful to incorporate $P P F D, \mathrm{CO}_{2}$ concentration, temperature, and their interactions into a single equation to estimate how changing multiple greenhouse environmental parameters will affect $\mathrm{P}_{\mathrm{n}}$. Cannell and Thornley (1998) and Körner et al. (2009) have shown clear interactive effects between these parameters. Cannell and Thornley (1998) developed a mathematical model to calculate gross photosynthetic rate as a function of temperature, $\mathrm{CO}_{2}$ concentration, and PPFD. They did so by developing relationships for quantum yield $(\alpha)$ and maximum gross photosynthetic rate $\left(\mathrm{P}_{\max }\right)$ as functions of $\mathrm{CO}_{2}$ concentration and temperature. These parameters, along with a fitting parameter, $\theta$, characterized the rate of gross photosynthesis in response to $P P F D$ using a nonrectangular hyperbolic function. Although the model developed by Cannell and Thornley (1998), which we will refer to as the Cannell and Thornley model, provides great conceptual value, the generic parameter values included in the original publication were not meant to accurately predict photosynthetic rate for any specific plant species.

The growth environment before measurement is another important factor affecting $\mathrm{P}_{\mathrm{n}}$, especially $P P F D$ and air temperature. Plants grown at higher PPFDs often, but not always, have higher photosynthetic capacities (Bunce and Heichel, 1986). The temperature optimum $\left(\mathrm{T}_{\mathrm{opt}}\right)$ of plants grown at higher temperatures is often higher than plants grown at lower temperatures (Berry and Björkman, 1980).

Therefore, the objectives of our study were to 1) measure the combined effects of light intensity and $\mathrm{CO}_{2}$ concentration on $\mathrm{P}_{\mathrm{n}}$ of plants grown at different $P P F D$ s; 2) measure the combined effects of $P P F D$, leaf temperature, and $\mathrm{CO}_{2}$ concentration on $\mathrm{P}_{\mathrm{n}}$ of plants grown at different air temperatures; and 3) fit the data to model $\mathrm{P}_{\mathrm{n}}$ as a function of leaf temperature, $P P F D$, and $\mathrm{CO}_{2}$ concentration.

\section{Materials and Methods}

\section{Plant care and treatments}

ExPT. 1. Calibrachoa (Calibrachoa $\times$ hybrida 'Superbells Lemon Slice'), petunia (Petunia $\times$ hybrida 'Supertunia Mini
Strawberry Pink Veined'), and verbena (Verbena $\times$ hybrida 'Superbena Royale Whitecap') cuttings (Pleasant View Gardens, Loudon, NH) in 84-cell count trays were trimmed and transplanted into 11.4-cm-diameter pots filled with soilless substrate (LC1; Sun Gro Horticulture, Bellevue, WA) and grown for $14 \mathrm{~d}$ in a growth chamber before the start of treatments. The PPFD was $\approx 270$ to $300 \mu \mathrm{mol} \cdot \mathrm{m}^{-2} \cdot \mathrm{s}^{-1}$, the photoperiod was $14 \mathrm{~h}$, and air temperature was $22 / 18^{\circ} \mathrm{C}$ day/night. Plants were watered as needed and fertilized at every irrigation with $20 \mathrm{~N}-4.4 \mathrm{P}-16.6 \mathrm{~K}$ (Jack's 20-10-20; JR Peters, Allentown, PA) at $150 \mathrm{mg} \cdot \mathrm{L}^{-1} \mathrm{~N}$.

After $14 \mathrm{~d}$, six plants of each species were moved to growth chambers that provided a PPFD of 90,180 , or $270 \mu \mathrm{mol} \cdot \mathrm{m}^{-2} \cdot \mathrm{s}^{-1}$ from cool-white fluorescent lamps. The photoperiod was $16 \mathrm{~h}$, and daily light integrals (DLIs) were 5.2, 10.4, and $15.6 \mathrm{~mol} \cdot \mathrm{m}^{-2} \cdot \mathrm{d}^{-1}$, respectively. Air temperature remained at $22 / 18^{\circ} \mathrm{C}$ day/night. Flowers were removed daily to minimize shading of developing leaves. Photosynthetic measurements commenced after $20 \mathrm{~d}$ (verbena), $28 \mathrm{~d}$ (petunia), and $42 \mathrm{~d}$ (calibrachoa) in the lighting treatments. Each crop required a different length of time for leaves initiated after plants were moved into the lighting treatments to expand enough to cover the measuring area of the instrument cuvette (described as follows).

Expт. 2. Geranium (Pelargonium $\times$ hortorum 'Maverick Red'), pepper (Capsicum annuum 'California Wonder'), and sunflower (Helianthus annuus 'Pacino Gold') (Ball Seed Co., West Chicago, IL) were sown in 288-cell count trays filled with soilless substrate (LC1). Seedlings were initially watered with reverse-osmosis water until true leaves emerged, at which point they were fertigated at every irrigation with $20 \mathrm{~N}-4.4 \mathrm{P}-16.6 \mathrm{~K}$ at $75 \mathrm{mg} \cdot \mathrm{L}^{-1} \mathrm{~N}$. Initial PPFD in the growth chamber was $\approx 150 \mu \mathrm{mol} \cdot \mathrm{m}^{-2} \cdot \mathrm{s}^{-1}$, the photoperiod was $12 \mathrm{~h}$, and air temperature was $22 / 18^{\circ} \mathrm{C}$ day/night. Sunflower and geranium were transplanted $24 \mathrm{~d}$ after sowing, and pepper was transplanted $31 \mathrm{~d}$ after sowing into 11.4-cm-diameter pots filled with soilless substrate (LC1). After transplant, PPFD was increased to $250 \mu \mathrm{mol} \cdot \mathrm{m}^{-2} \cdot \mathrm{s}^{-1}$, photoperiod was increased to $14 \mathrm{~h}$, and the nutrient solution concentration was increased to $150 \mathrm{mg} \cdot \mathrm{L}^{-1} \mathrm{~N}$.

Three (pepper) or four (sunflower and geranium) weeks after transplant, seven plants of each species were moved to growth chambers set at constant air temperatures of 14,21 , or $28^{\circ} \mathrm{C}$. The PPFD remained at $250 \mu \mathrm{mol} \cdot \mathrm{m}^{-2} \cdot \mathrm{s}^{-1}$ and the photoperiod was increased to $16 \mathrm{~h}$, resulting in a DLI of $14.4 \mathrm{~mol} \cdot \mathrm{m}^{-2} \cdot \mathrm{d}^{-1}$. Photosynthetic measurements commenced after $14 \mathrm{~d}$ (sunflower), $21 \mathrm{~d}$ (pepper), and $29 \mathrm{~d}$ (geranium) in these treatments. As noted previously, each crop required a different length of time for leaves initiated after plants were moved into temperature treatments to expand enough for photosynthetic measurements.

\section{Photosynthetic measurements}

In both experiments, $P_{n}$ was measured once during production for each crop and treatment combination, and all plants in a crop were evaluated before proceeding to the next crop. Five plants per species per treatment (DLI or temperature) were selected in random order for measurement. A single leaf from each plant was used. Recently emerged, fully expanded, unshaded leaves were chosen whenever possible. However, recently emerged leaves on calibrachoa and petunia were often too small to cover the measurement area, and this occurred in all three DLI treatments in Expt 1. In those instances, larger, but not necessarily recently emerged, leaves were selected instead. In Expt. 2, geraniums grown at $28^{\circ} \mathrm{C}$ exhibited high temperatureinduced leaf yellowing and bleaching on leaves that emerged 
while at $28^{\circ} \mathrm{C}$. Preliminary tests on the yellowed leaves resulted in negligible $\mathrm{P}_{\mathrm{n}}$, therefore, older (greener) leaves of geranium grown at $28^{\circ} \mathrm{C}$ were used instead.

Photosynthetic response curve measurements were collected using a portable photosynthesis system (LI-6400XT; LI-COR Biosciences, Lincoln, NE). Leaf temperature, flow rate, PPFD, and reference $\mathrm{CO}_{2}$ concentration were precisely controlled in a leaf chamber. A cuvette with a $6-\mathrm{cm}^{2}$ area and red and blue light-emitting diodes [LEDs (6400-02B, LI-COR Biosciences)] was used for most species. A cuvette with a $2-\mathrm{cm}^{2}$ measurement area (6400-40, LI-COR Biosciences) was used for calibrachoa and petunia because of their small leaf size. The LI-6400XT reports $\mathrm{P}_{\mathrm{n}}$ on a leaf area basis and automatically accounts for a change in measurement area when using cuvettes with different apertures. Both LED sources emitted a red:blue light ratio of 90:10. Leaf temperature was maintained at ambient growth chamber air temperature $\left(22^{\circ} \mathrm{C}\right.$ for calibrachoa, petunia, and verbena, and 14,21 , or $28^{\circ} \mathrm{C}$ for geranium, pepper, and sunflower).

Measurements were collected using an autoprogram that cycled through a predetermined sequence of conditions, logging $\mathrm{P}_{\mathrm{n}}$ at each step once stability parameters were attained. The leaf was placed in the measurement chamber and acclimated to initial conditions of $400 \mu \mathrm{mol} \cdot \mathrm{mol}^{-1} \mathrm{CO}_{2}$ and $2000 \mu \mathrm{mol} \cdot \mathrm{m}^{-2} \cdot \mathrm{s}^{-1}$ $P P F D$. Net photosynthesis was then measured at 14 light intensities, ranging from 2000 to $0 \mu \mathrm{mol} \cdot \mathrm{m}^{-2} \cdot \mathrm{s}^{-1}$, and four reference $\mathrm{CO}_{2}$ concentrations $\left(200,400,600\right.$, and $\left.800 \mu \mathrm{mol} \cdot \mathrm{mol}^{-1}\right)$. For each leaf, each light response curve was measured at 400 $\mu \mathrm{mol} \cdot \mathrm{mol}^{-1} \mathrm{CO}_{2}$, then 200, 600, and $800 \mu \mathrm{mol} \cdot \mathrm{mol}^{-1} \mathrm{CO}_{2}$. Once the leaf was acclimated, measurements were collected in descending PPFD $(2000,1500,1000,800,700,600,500,400$, $300,200,100,50,25$, and $\left.0 \mu \mathrm{mol} \cdot \mathrm{m}^{-2} \cdot \mathrm{s}^{-1}\right)$. An adjustment period of at least $5 \mathrm{~min}$ was programmed at $2000 \mu \mathrm{mol} \cdot \mathrm{m}^{-2} \cdot \mathrm{s}^{-1}$ $P P F D$ between each light response curve at each $\mathrm{CO}_{2}$ concentration, and in addition, two consecutive $\mathrm{P}_{\mathrm{n}}$ measurements were taken at $2000 \mu \mathrm{mol} \cdot \mathrm{m}^{-2} \cdot \mathrm{s}^{-1}$ PPFD to verify that $\mathrm{P}_{\mathrm{n}}$ had indeed stabilized. Measurements were logged once stability parameters were attained (minimum and maximum wait times of 45 and 90 $\mathrm{s}$, respectively, were defined when using the $6-\mathrm{cm}^{2}$ cuvette, and 60 and $90 \mathrm{~s}$, respectively, when using the $2-\mathrm{cm}^{2}$ cuvette). Stability criteria included the rate of change for $\mathrm{P}_{\mathrm{n}}$, reference $\mathrm{CO}_{2}$ concentration, reference $\mathrm{H}_{2} \mathrm{O}$ concentration, and flow rate.

\section{Data analysis}

For each plant, $\mathrm{P}_{\mathrm{n}}$ light response curves were fit at each $\mathrm{CO}_{2}$ concentration using the nonrectangular hyperbola (NRH) function presented in Eq. [1]. It is a common model for fitting photosynthetic response curves to empirical data (e.g., Boldt et al., 2014; Cannell and Thornley, 1998), and the parameter estimates can be related to photosynthetic characteristics of the plant:

$$
\mathrm{P}_{\mathrm{n}}=1 / 2 \theta \times\left\{\alpha \mathrm{I}+\mathrm{P}_{\max }-\left[\left(\alpha \mathrm{I}+\mathrm{P}_{\max }\right)^{2}-4 \alpha \theta \mathrm{P}_{\max } \mathrm{I}\right]^{0.5}\right\}-\mathrm{R}_{\mathrm{d}},
$$

where $\mathrm{P}_{\mathrm{n}}=$ net photosynthetic rate (micromoles $\mathrm{CO}_{2}$ per square meter per second), $\theta=$ curvature parameter (value constrained between 0 and 1), $\alpha=$ initial quantum efficiency (micromoles $\mathrm{CO}_{2}$ per micromole photons), $\mathrm{P}_{\max }=$ maximum (gross) photosynthetic rate (micromoles $\mathrm{CO}_{2}$ per square meter per second), $\mathrm{R}_{\mathrm{d}}=$ respiration (micromoles $\mathrm{CO}_{2}$ per square meter per second), and $\mathrm{I}=$ irradiance (micromoles per square meter per second).
Parameters in Eq. [1] $\left(\mathrm{P}_{\max }, \alpha, \mathrm{R}_{\mathrm{d}}\right.$, and $\left.\theta\right)$ were fit by minimizing the sum of squares between the data points and the values modeled for measurements taken at each growth environment [GE (DLI for Expt. 1 and temperature for Expt. 2)] and $\mathrm{CO}_{2}$ concentration combination. Data from each light response curve were fit independently. Regression was performed using the Ipopt solver [version 0.2.6 (Wächter and Biegler, 2006)] in the JuMP modeling language [version 0.17.1 (Dunning et al., 2017)] written in the Julia programming language [version 0.5.2 (Bezanson et al., 2012)].

The light saturation point (LSP) and light compensation point (LCP) were calculated for each light response curve. The LSP was defined as the light intensity at which $\mathrm{P}_{\mathrm{n}}$ equaled $90 \%$ of the maximum net photosynthetic rate $P_{n, \max }\left(P_{\max }-R_{d}\right)$, as previously defined (Jurik et al., 1988; Ronquim et al., 2009). The LCP was the light intensity at which $\mathrm{P}_{\mathrm{n}}$ equaled zero.

Analysis of variance was conducted in $\mathrm{R}$ (version 3.3.1; R Foundation for Statistical Computing, Vienna, Austria) on the NRH parameter values within each species and GE combination. Where significant $(P<0.05)$, Tukey's honestly significant difference test $(\alpha=0.05)$ was used for mean separation.

Data in Expt. 2 were also fit to a model developed by Cannell and Thornley (1998), which defined $\alpha$ and $\mathrm{P}_{\max }$ (as defined in Eq. [1]) as functions of temperature and $\mathrm{CO}_{2}$ concentration, as shown in Eq. [2] through Eq. [9]. They modeled gross photosynthesis, but by including $\mathrm{R}_{\mathrm{d}}$ in Eq. 1 , we modeled $\mathrm{P}_{\mathrm{n}}$ instead. In addition, we modified the reference $\mathrm{CO}_{2}$ concentration from 350 to $400 \mu \mathrm{mol} \cdot \mathrm{mol}^{-1}$ to reflect recent increases in atmospheric $\mathrm{CO}_{2}$ concentration, and we modified the reference temperature for $\alpha\left(\mathrm{T}_{\text {ref }, \alpha}\right)$ from 15 to $14^{\circ} \mathrm{C}$ to allow our data to be modeled using a continuous function. At temperatures below $\mathrm{T}_{\text {ref }}$, Cannell and Thornley (1998) assumed the value of $\alpha$ was constant in relation to temperature.

The value of $\alpha$ is modeled by Eq. [2] to Eq. [4]:

$$
\alpha=\alpha_{\text {ref }} \times f_{C a, \alpha} \times f_{T, \alpha},
$$

where $\alpha_{\text {ref }}=$ the value of $\alpha$ at $\mathrm{T}_{\text {ref, } \alpha}$ and $400 \mu \mathrm{mol} \cdot \mathrm{mol}^{-1} \mathrm{CO}_{2}$, $f_{C a, \alpha}=$ a factor representing the influence of $\mathrm{CO}_{2}$ concentration on $\alpha$, and $f_{T, \alpha}=$ a factor representing the influence of temperature on $\alpha$.

$$
\begin{gathered}
f_{C a, \alpha}=1-\beta / \mathrm{C}_{\mathrm{a}} \\
f_{T, \alpha}=1-\mathrm{c}_{\mathrm{T}, \alpha} \times\left(\mathrm{T}_{\text {leaf }}-\mathrm{T}_{\text {ref }, \alpha}\right) \times 400 / \mathrm{C}_{\mathrm{a}}
\end{gathered}
$$

where $\beta=$ a coefficient representing the sensitivity of $f_{\mathrm{Ca}, \alpha}$ to $\mathrm{CO}_{2}$ concentration (micromoles per mole), $\mathrm{C}_{\mathrm{a}}=\mathrm{CO}_{2}$ concentration (micromoles per mole), and $\mathrm{c}_{\mathrm{T}, \alpha}=\mathrm{a}$ coefficient representing the sensitivity of $f_{T, \alpha}$ to temperature $\left({ }^{\circ} \mathrm{C}^{-1}\right)$, $\mathrm{T}_{\text {leaf }}=$ leaf temperature, and $\mathrm{T}_{\text {ref, }, \alpha}=$ the reference temperature for $\alpha$. The inclusion of $\mathrm{CO}_{2}$ concentration in Eq. [4] modifies the sensitivity of $\alpha$ to temperature based on $\mathrm{CO}_{2}$ concentration.

The value of $\mathrm{P}_{\max }$ is modeled by Eq. [5] to Eq. [9]:

$$
\mathrm{P}_{\max }=\mathrm{P}_{\max , \text { ref }} \times f_{C a, P \max } \times f_{T, P \max }
$$

where $P_{\text {max,ref }}=$ the value of $P_{\max }$ at a reference temperature and $\mathrm{CO}_{2}$ concentration (assumed to be $20^{\circ} \mathrm{C}$ and $400 \mu \mathrm{mol} \cdot \mathrm{mol}^{-1}$ ), $f_{C a, P \max }=\mathrm{a}$ factor representing the influence of $\mathrm{CO}_{2}$ concentration on $\mathrm{P}_{\max }$, and $f_{T, P \max }=\mathrm{a}$ factor representing the influence of temperature on $\mathrm{P}_{\max }$.

$$
f_{C a, P \max }=1 /\left(1+\mathrm{K}_{\mathrm{Ca}, \mathrm{Pmax}} / \mathrm{C}_{\mathrm{a}}\right)
$$




$$
\begin{aligned}
f_{T, P \max }= & {\left[\left(\mathrm{T}-\mathrm{T}_{0}\right) /\left(\mathrm{T}_{\text {ref }}-\mathrm{T}_{0}\right)\right]^{2} } \\
& \times\left[\left(\mathrm{T}_{0, \text { Pmax }}-\mathrm{T}\right) /\left(\mathrm{T}_{0, \text { Pmax }}-\mathrm{T}_{\text {ref }}\right)\right]
\end{aligned}
$$

where $\mathrm{K}_{\mathrm{Ca}, \mathrm{Pmax}}=\mathrm{a}$ coefficient representing the sensitivity of $f_{C a, P \max }$ to $\mathrm{CO}_{2}$ concentration (micromoles per mole), $\mathrm{T}_{0}=$ the lower temperature at which $f_{T, P \max }$ is $0\left({ }^{\circ} \mathrm{C}\right)$, and $\mathrm{T}_{0, P \max }=$ the upper temperature at which $f_{T, P \max }$ is $0\left({ }^{\circ} \mathrm{C}\right)$.

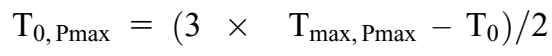

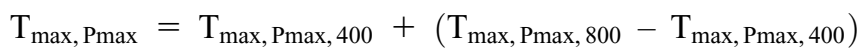

$$
\times\left(\mathrm{C}_{\mathrm{a}}-400\right) /(800-400)
$$

where $\mathrm{T}_{\max , \mathrm{Pmax}, 400}=$ the temperature $\left({ }^{\circ} \mathrm{C}\right)$ that maximizes $\mathrm{P}_{\max }$ at $400 \mu \mathrm{mol} \cdot \mathrm{mol}^{-1} \mathrm{CO}_{2}$ concentration and $\mathrm{T}_{\max , \mathrm{Pmax}, 800}=$ the temperature $\left({ }^{\circ} \mathrm{C}\right)$ that maximizes $\mathrm{P}_{\max }$ at $800 \mu \mathrm{mol} \cdot \mathrm{mol}^{-1} \mathrm{CO}_{2}$ concentration.

To fit the general model developed by Cannell and Thornley (1998) with the addition of the parameter $R_{d}$, the parameters $\alpha_{\text {ref }}, \beta, \theta, c_{T, \alpha}, P_{\text {max }, \text { ref }}, K_{\text {Ca,Pmax }}, T_{\text {max }, P \max , 400}, T_{\text {max }, P \max , 800}, T_{0}$, and $R_{d}$ were determined using least squares regression to minimize differences between data for each species and the Cannell and Thornley model. Regression was performed as previously described for individual response curves.

\section{Results}

ExPT. 1. Verbena and petunia acclimated to higher light intensities (180 and $\left.270 \mu \mathrm{mol} \cdot \mathrm{m}^{-2} \cdot \mathrm{s}^{-1} P P F D\right)$ had higher rates of $\mathrm{P}_{\max }$ compared with plants acclimated to $90 \mu \mathrm{mol} \cdot \mathrm{m}^{-2} \cdot \mathrm{s}^{-1}$ PPFD (Fig. 1). In verbena, $\mathrm{P}_{\max }$ ranged from 8.6 to 25.1 $\mu \mathrm{mol} \cdot \mathrm{m}^{-2} \cdot \mathrm{s}^{-1} \mathrm{CO}_{2}$ for plants grown at $90 \mu \mathrm{mol} \cdot \mathrm{m}^{-2} \cdot \mathrm{s}^{-1} P P F D$, depending on $\mathrm{CO}_{2}$ concentration, but increased to 11.2 to 33.4 $\mu \mathrm{mol} \cdot \mathrm{m}^{-2} \cdot \mathrm{s}^{-1} \mathrm{CO}_{2}$ and 12.2 to $33.0 \mu \mathrm{mol} \cdot \mathrm{m}^{-2} \cdot \mathrm{s}^{-1} \mathrm{CO}_{2}$ for plants grown at 180 and $270 \mu \mathrm{mol} \cdot \mathrm{m}^{-2} \cdot \mathrm{s}^{-1} P P F D$, respectively (Table 1). In petunia, $P_{\max }$ ranged from 6.9 to 17.9 $\mu \mathrm{mol} \cdot \mathrm{m}^{-2} \cdot \mathrm{s}^{-1} \mathrm{CO}_{2}, 10.3$ to $28.5 \mu \mathrm{mol} \cdot \mathrm{m}^{-2} \cdot \mathrm{s}^{-1} \mathrm{CO}_{2}$, and 9.1 to $27.1 \mu \mathrm{mol} \cdot \mathrm{m}^{-2} \cdot \mathrm{s}^{-1} \mathrm{CO}_{2}$ for plants grown at 90,180 , and 270 $\mu \mathrm{mol} \cdot \mathrm{m}^{-2} \cdot \mathrm{s}^{-1} P P F D$, respectively (Table 1 ). Results for calibrachoa were inconsistent between treatments, with the highest values of $\mathrm{P}_{\max }\left(6.4\right.$ to $17.4 \mu \mathrm{mol} \cdot \mathrm{m}^{-2} \cdot \mathrm{s}^{-1} \mathrm{CO}_{2}$ ) occurring in plants grown at $90 \mu \mathrm{mol} \cdot \mathrm{m}^{-2} \cdot \mathrm{s}^{-1}$ PPFD and the lowest values of $\mathrm{P}_{\max }$

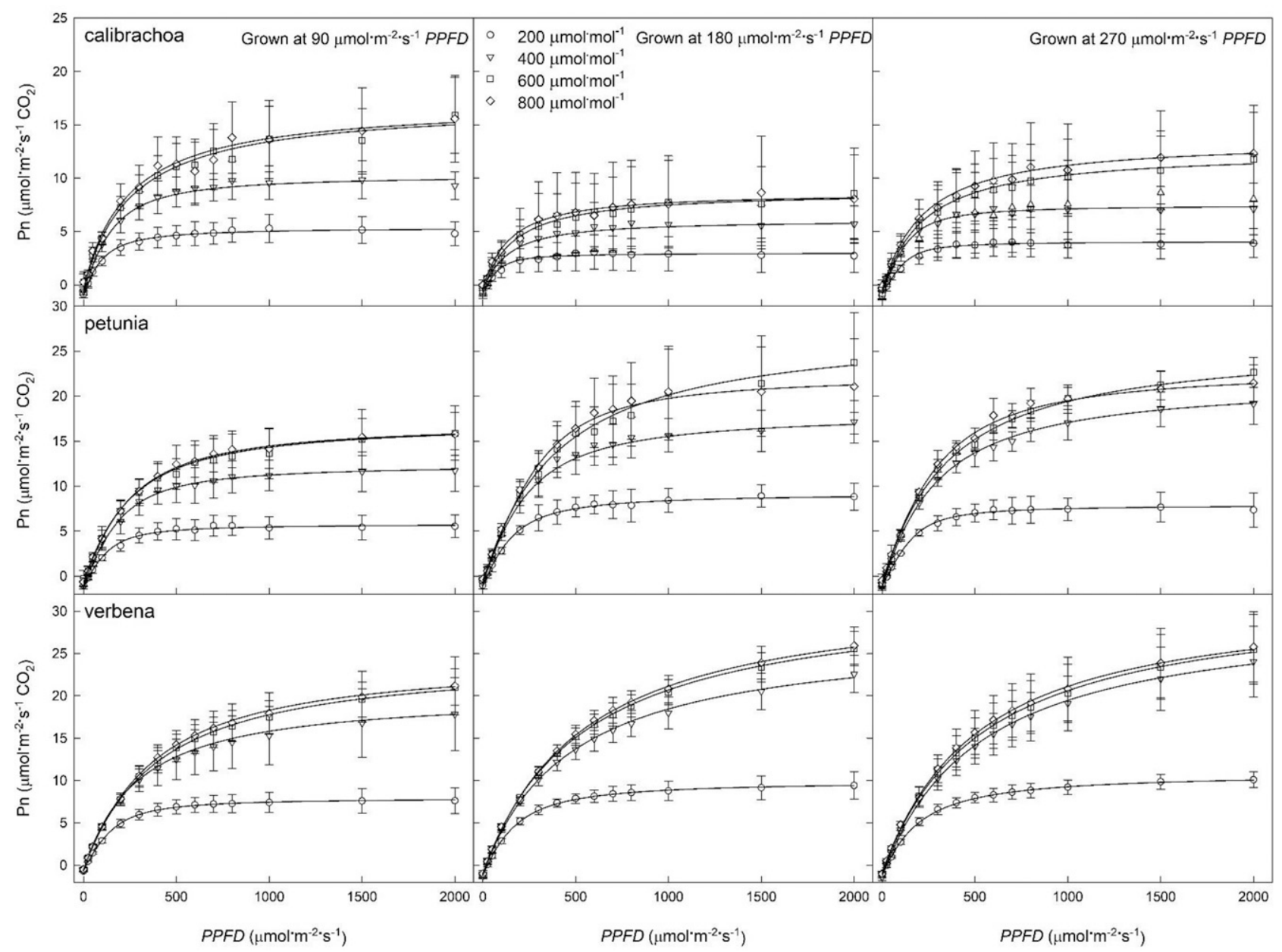

Fig. 1. Net photosynthesis $\left(\mathrm{P}_{\mathrm{n}}\right)$ of calibrachoa 'Superbells Lemon Slice', petunia 'Supertunia Mini Strawberry Pink Veined', and verbena 'Superbena Royale Whitecap' (top, middle, and bottom rows, respectively) grown at photosynthetic photon flux densities (PPFDs) of 90,180 , and $270 \mu \mathrm{mol} \cdot \mathrm{m}^{-2} \cdot \mathrm{s}^{-1}$ (left, center, and right columns, respectively). Plants were measured at $14 P P F D \mathrm{~s}$ and four $\mathrm{CO}_{2}$ concentrations. Points plotted are the means $( \pm \mathrm{SD})$ of each treatment $(\mathrm{n}=5)$ at each $P P F D$. Lines are the modeled values using the equation $\mathrm{P}_{\mathrm{n}}=1 / 2 \theta\left\{\alpha \mathrm{I}+\mathrm{P}_{\max }-\left[\left(\alpha \mathrm{I}+\mathrm{P}_{\max }\right)^{2}-4 \alpha \theta \mathrm{P}_{\max } \cdot \mathrm{I}\right]^{0.5}\right\}-\mathrm{R}_{\mathrm{d}}$, with the parameter values listed in Table 1. 
Table 1. Nonrectangular hyperbola equation parameters [maximum photosynthetic rate $\left(\mathrm{P}_{\max }\right)$, quantum yield $(\alpha)$, $\theta$, and dark respiration $\left(\mathrm{R}_{\mathrm{d}}\right)$ ] for photosynthetic light response curves of calibrachoa, petunia, and verbena grown at three photosynthetic photon flux densities $(90$, 180 , and $\left.270 \mu \mathrm{mol} \cdot \mathrm{m}^{-2} \cdot \mathrm{s}^{-1} P P F D\right)$ and measured at four $\mathrm{CO}_{2}$ concentrations $\left(200,400,600\right.$, and $\left.800 \mu \mathrm{mol} \cdot \mathrm{mol}^{-1}\right)$. The light compensation point $\left\{\mathrm{LCP}\left[P P F D\right.\right.$ at which net photosynthesis $\left.\left.\left(\mathrm{P}_{\mathrm{n}}\right)=0\right]\right\}$ and light saturation point [LSP (PPFD corresponding to 90\% net $\left.\mathrm{P}_{\text {max }}\right)$ ] were calculated from the nonrectangular hyperbola, $\mathrm{P}_{\mathrm{n}}=1 / 2 \theta\left\{\alpha \mathrm{I}+\mathrm{P}_{\max }-\left[\left(\alpha \mathrm{I}+\mathrm{P}_{\max }\right)^{2}-4 \alpha \theta \mathrm{P}_{\max } \mathrm{I}\right]^{0.5}\right\}-\mathrm{R}_{\mathrm{d}}$. All fitted parameters and calculated values are mean \pm SD $(n=5)$.

\begin{tabular}{|c|c|c|c|c|c|c|c|}
\hline \multirow[b]{2}{*}{$\mathrm{GE} / \mathrm{CO}_{2}{ }^{\mathrm{z}}$} & $\begin{array}{c}\mathrm{P}_{\max } \\
\left(\mu \mathrm{mol} \cdot \mathrm{m}^{-2} \cdot \mathrm{s}^{-1}\right. \\
\left.\mathrm{CO}_{2}\right) \\
\end{array}$ & $\begin{array}{c}\alpha\left[\mathrm{CO}_{2}\right. \\
\left(\mu \mathrm{mol}^{\prime} \mu \mathrm{mol}^{-1}\right. \\
\text { photons })] \\
\end{array}$ & $\theta$ & $\begin{array}{c}\mathrm{R}_{\mathrm{d}} \\
\left(\mu \mathrm{mol} \cdot \mathrm{m}^{-2} \cdot \mathrm{s}^{-1}\right. \\
\left.\mathrm{CO}_{2}\right) \\
\end{array}$ & $\begin{array}{c}\mathrm{LSP} \\
\left(\mu \mathrm{mol} \cdot \mathrm{m}^{-2} \cdot \mathrm{s}^{-1}\right) \\
\end{array}$ & $\begin{array}{c}\mathrm{LCP} \\
\left(\mu \mathrm{mol} \cdot \mathrm{m}^{-2} \cdot \mathrm{s}^{-1}\right) \\
\end{array}$ & \multirow{2}{*}{$\begin{array}{c}\text { Pseudo } R^{2} \\
\left(1-\mathrm{SS}_{\text {error }} / \mathrm{SS}_{\text {total }}\right)^{\mathrm{y}}\end{array}$} \\
\hline & \multicolumn{6}{|c|}{$($ mean $\pm \mathrm{SD})$} & \\
\hline \multicolumn{8}{|c|}{ Calibrachoa 'Lemon Slice' } \\
\hline $90 / 200$ & $6.4 \pm 0.9 \mathrm{~b}^{\mathrm{x}}$ & $0.0510 \pm 0.0072 \mathrm{c}$ & $0.533 \pm 0.045 \mathrm{a}$ & $0.98 \pm 0.40$ & $709 \pm 110$ & $20.8 \pm 7.2 \mathrm{a}$ & 0.843 \\
\hline $90 / 400$ & $11.0 \pm 1.3 \mathrm{~b}$ & $0.0550 \pm 0.0031 \mathrm{bc}$ & $0.593 \pm 0.347 \mathrm{a}$ & $0.64 \pm 0.39$ & $873 \pm 542$ & $11.9 \pm 7.2 \mathrm{ab}$ & 0.895 \\
\hline $90 / 600$ & $17.4 \pm 3.8 \mathrm{a}$ & $0.0670 \pm 0.0043 \mathrm{ab}$ & $0.001 \pm 0.000 \mathrm{~b}$ & $0.37 \pm 0.34$ & $>2000^{\mathrm{w}}$ & $5.5 \pm 5.1 \mathrm{~b}$ & 0.863 \\
\hline $90 / 800$ & $17.4 \pm 4.9 \mathrm{a}$ & $0.0727 \pm 0.0097 \mathrm{a}$ & $0.014 \pm 0.028 b$ & $0.37 \pm 0.34$ & $>2000$ & $4.9 \pm 4.6 b$ & 0.800 \\
\hline ANOVA & $<0.001$ & $<0.001$ & $<0.001$ & 0.0574 & - & 0.003 & \\
\hline $\operatorname{HSD}^{\mathrm{x}}$ & 5.8 & 0.0120 & 0.318 & - & - & 11.1 & \\
\hline $180 / 200$ & $3.8 \pm 1.9$ & $0.0361 \pm 0.0194$ & $0.733 \pm 0.364 \mathrm{a}$ & $0.81 \pm 0.43$ & $359 \pm 193 b$ & $26.8 \pm 11.2 \mathrm{a}$ & 0.515 \\
\hline $180 / 400$ & $6.6 \pm 2.3$ & $0.0418 \pm 0.0099$ & $0.329 \pm 0.459 \mathrm{ab}$ & $0.47 \pm 0.43$ & $974 \pm 619 a b$ & $11.7 \pm 10.4 \mathrm{ab}$ & 0.635 \\
\hline $180 / 600$ & $9.4 \pm 5.0$ & $0.0561 \pm 0.0267$ & $0.054 \pm 0.118 \mathrm{~b}$ & $0.63 \pm 0.61$ & $1504 \pm 504 \mathrm{a}$ & $9.8 \pm 7.0 \mathrm{~b}$ & 0.505 \\
\hline $180 / 800$ & $9.0 \pm 5.0$ & $0.0567 \pm 0.0123$ & $0.223 \pm 0.333 \mathrm{ab}$ & $0.29 \pm 0.29$ & $1047 \pm 506 \mathrm{ab}$ & $5.3 \pm 5.1 \mathrm{~b}$ & 0.452 \\
\hline ANOVA & 0.12 & 0.23 & 0.038 & 0.34 & 0.015 & 0.008 & \\
\hline HSD & - & - & 0.619 & - & 873 & 15.9 & \\
\hline $270 / 200$ & $5.0 \pm 1.4 \mathrm{~b}$ & $0.0307 \pm 0.0077 \mathrm{~b}$ & $0.833 \pm 0.084 \mathrm{a}$ & $0.89 \pm 0.50$ & $436 \pm 153 b$ & $28.9 \pm 11.2 \mathrm{a}$ & 0.745 \\
\hline $270 / 400$ & $8.3 \pm 2.5 \mathrm{ab}$ & $0.0465 \pm 0.0095 \mathrm{ab}$ & $0.757 \pm 0.245 \mathrm{a}$ & $0.77 \pm 0.48$ & $553 \pm 382 b$ & $16.9 \pm 9.6 \mathrm{ab}$ & 0.737 \\
\hline $270 / 600$ & $13.4 \pm 5.0 \mathrm{a}$ & $0.0596 \pm 0.0129 \mathrm{a}$ & $0.092 \pm 0.150 \mathrm{~b}$ & $0.77 \pm 0.45$ & $1915 \pm 522 \mathrm{a}$ & $13.0 \pm 6.0 \mathrm{~b}$ & 0.725 \\
\hline $270 / 800$ & $14.1 \pm 5.5 \mathrm{a}$ & $0.0601 \pm 0.0077 \mathrm{a}$ & $0.143 \pm 0.203 \mathrm{~b}$ & $0.50 \pm 0.39$ & $1863 \pm 639 a$ & $8.3 \pm 5.5 b$ & 0.698 \\
\hline ANOVA & 0.007 & $<0.001$ & $<0.001$ & 0.599 & $<0.001$ & 0.009 & \\
\hline HSD & 7.3 & 0.0176 & 0.327 & - & 834 & 15.3 & \\
\hline \multicolumn{8}{|c|}{ Petunia 'Supertunia Mini Strawberry Pink Veined' } \\
\hline $90 / 200$ & $6.9 \pm 1.4 \mathrm{~b}$ & $0.0392 \pm 0.0163$ & $0.747 \pm 0.185$ & $1.12 \pm 0.45$ & $607 \pm 228 \mathrm{~b}$ & $30.7 \pm 6.0 \mathrm{a}$ & 0.872 \\
\hline $90 / 400$ & $13.5 \pm 2.3 \mathrm{a}$ & $0.0523 \pm 0.0088$ & $0.670 \pm 0.168$ & $1.05 \pm 0.36$ & $1034 \pm 486 \mathrm{ab}$ & $20.4 \pm 5.5 \mathrm{ab}$ & 0.903 \\
\hline $90 / 600$ & $17.9 \pm 4.6 \mathrm{a}$ & $0.0598 \pm 0.0182$ & $0.421 \pm 0.286$ & $0.65 \pm 0.39$ & $1788 \pm 716$ a & $11.3 \pm 6.6 \mathrm{~b}$ & 0.892 \\
\hline $90 / 800$ & $17.9 \pm 3.3 \mathrm{a}$ & $0.0578 \pm 0.0177$ & $0.500 \pm 0.334$ & $0.73 \pm 0.53$ & $1592 \pm 714 \mathrm{ab}$ & $12.6 \pm 7.5 \mathrm{~b}$ & 0.929 \\
\hline ANOVA & $<0.001$ & 0.197 & 0.195 & 0.282 & 0.019 & $<.001$ & \\
\hline HSD & 5.7 & - & - & - & 1036 & 11.6 & \\
\hline $180 / 200$ & $10.3 \pm 1.5 \mathrm{~b}$ & $0.0497 \pm 0.0092$ & $0.618 \pm 0.170 \mathrm{ab}$ & $1.09 \pm 0.56$ & $918 \pm 301$ & $22.2 \pm 11.1 \mathrm{a}$ & 0.909 \\
\hline $180 / 400$ & $19.1 \pm 3.0 \mathrm{ab}$ & $0.0637 \pm 0.0145$ & $0.532 \pm 0.378 \mathrm{ab}$ & $0.88 \pm 0.54$ & $1441 \pm 876$ & $13.3 \pm 7.6 \mathrm{ab}$ & 0.930 \\
\hline $180 / 600$ & $28.5 \pm 8.1 \mathrm{a}$ & $0.0680 \pm 0.0139$ & $0.170 \pm 0.246 b$ & $0.72 \pm 0.47$ & $>2000$ & $10.1 \pm 5.2 \mathrm{ab}$ & 0.872 \\
\hline $180 / 800$ & $23.0 \pm 5.9 \mathrm{a}$ & $0.0560 \pm 0.0130$ & $0.759 \pm 0.245 \mathrm{a}$ & $0.45 \pm 0.45$ & $1443 \pm 702$ & $7.3 \pm 4.7 \mathrm{~b}$ & 0.874 \\
\hline ANOVA & $<0.001$ & 0.155 & 0.021 & 0.24 & - & 0.036 & \\
\hline HSD & 9.5 & - & 0.489 & - & - & 13.7 & \\
\hline $270 / 200$ & $9.1 \pm 2.1 \mathrm{~b}$ & $0.0422 \pm 0.0066 \mathrm{~b}$ & $0.806 \pm 0.105 \mathrm{a}$ & $1.15 \pm 0.33$ & $602 \pm 260$ & $27.6 \pm 5.2 \mathrm{a}$ & 0.905 \\
\hline $270 / 400$ & $23.1 \pm 2.9 \mathrm{a}$ & $0.0707 \pm 0.0093 \mathrm{a}$ & $0.280 \pm 0.188 \mathrm{~b}$ & $1.34 \pm 0.44$ & $>2000$ & $19.5 \pm 4.2 \mathrm{ab}$ & 0.971 \\
\hline $270 / 600$ & $27.1 \pm 3.2 \mathrm{a}$ & $0.0729 \pm 0.0179 \mathrm{a}$ & $0.176 \pm 0.357 \mathrm{~b}$ & $1.08 \pm 0.57$ & $>2000$ & $14.3 \pm 6.3 b$ & 0.985 \\
\hline $270 / 800$ & $23.9 \pm 3.1 \mathrm{a}$ & $0.0632 \pm 0.0155 \mathrm{ab}$ & $0.605 \pm 0.260 \mathrm{ab}$ & $0.64 \pm 0.61$ & $1554 \pm 694$ & $9.0 \pm 8.5 \mathrm{~b}$ & 0.973 \\
\hline ANOVA & $<0.001$ & 0.008 & 0.003 & 0.193 & - & 0.002 & \\
\hline HSD & 5.1 & 0.0237 & 0.445 & - & - & 11.3 & \\
\hline \multicolumn{8}{|c|}{ Verbena 'Superbena Royale Whitecap' } \\
\hline $90 / 200$ & $8.6 \pm 1.7 \mathrm{~b}$ & $0.0425 \pm 0.0086 \mathrm{~b}$ & $0.720 \pm 0.177 \mathrm{a}$ & $0.63 \pm 0.14$ & $687 \pm 300$ & $15.1 \pm 1.8 \mathrm{a}$ & 0.928 \\
\hline $90 / 400$ & $21.0 \pm 5.8 \mathrm{a}$ & $0.0694 \pm 0.0037 \mathrm{a}$ & $0.133 \pm 0.218 \mathrm{~b}$ & $0.68 \pm 0.17$ & $>2000$ & $10.1 \pm 2.4 \mathrm{~b}$ & 0.874 \\
\hline $90 / 600$ & $25.1 \pm 2.7 \mathrm{a}$ & $0.0601 \pm 0.0071 \mathrm{a}$ & $0.148 \pm 0.087 \mathrm{~b}$ & $0.53 \pm 0.22$ & $>2000$ & $8.9 \pm 3.5 b$ & 0.965 \\
\hline $90 / 800$ & $25.1 \pm 4.5 \mathrm{a}$ & $0.0592 \pm 0.0053 \mathrm{a}$ & $0.289 \pm 0.064 \mathrm{~b}$ & $0.53 \pm 0.15$ & $>2000$ & $9.0 \pm 2.2 \mathrm{~b}$ & 0.949 \\
\hline
\end{tabular}


Table 1. (Continued)

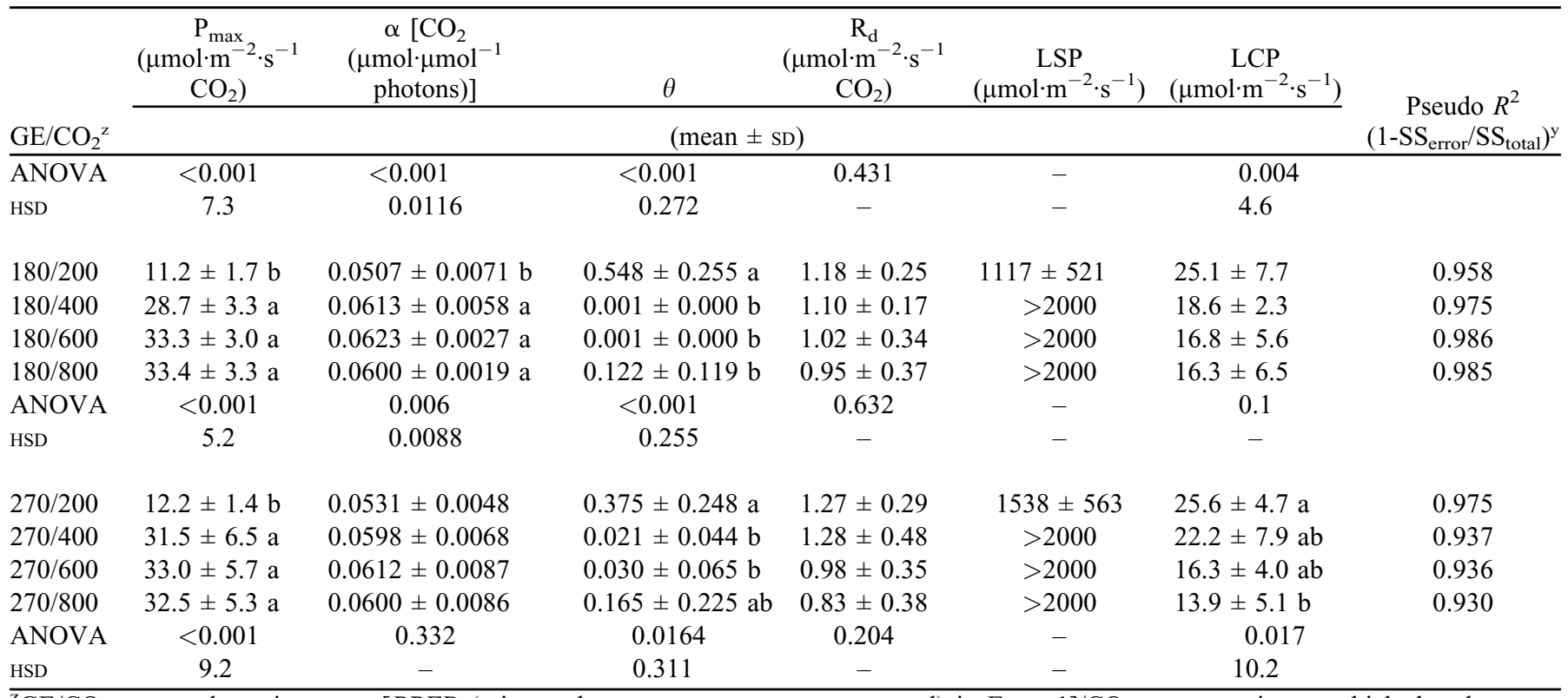

${ }^{\bar{z}} \mathrm{GE} / \mathrm{CO}_{2}=$ growth environment $\left[P P F D\right.$ (micromoles per square meter per second) in Expt. 1]/CO ${ }_{2}$ concentration at which the photosynthetic light response curve was determined.

${ }^{\mathrm{y}} \mathrm{SS}_{\text {error }} / \mathrm{SS}_{\text {total }}=$ sum of squares (error)/sum of squares (total).

${ }^{\mathrm{x}}$ Tukey's honestly significant difference (HSD) at $\alpha=0.05$. A hyphen for HSD indicates test not performed $(P>0.05)$. Within each species and growth environment $(\mathrm{GE}) / \mathrm{CO}_{2}$, means followed by similar letters are not statistically different at $\alpha=0.05$.

${ }^{\mathrm{w}}$ LSP values exceeding the upper bounds evaluated are noted as $>2000 \mu \mathrm{mol} \cdot \mathrm{m}^{-2} \cdot \mathrm{s}^{-1} P P F D$ rather than reporting an extrapolated value; analysis of variance (ANOVA) not performed for the GE.

(3.8 to $9.4 \mu \mathrm{mol} \cdot \mathrm{m}^{-2} \cdot \mathrm{s}^{-1} \mathrm{CO}_{2}$ ) occurring in plants acclimated to $180 \mu \mathrm{mol} \cdot \mathrm{m}^{-2} \cdot \mathrm{s}^{-1}$ PPFD (Fig. 1, Table 1 ).

In all three species, $\mathrm{P}_{\max }$ generally increased with increasing $\mathrm{CO}_{2}$ concentration, although the magnitude of increase was generally less with higher $\mathrm{CO}_{2}$ concentrations. For example, $\mathrm{P}_{\max }$ of verbena increased $144 \%, 156 \%$, and $158 \%$ from 200 to 400 $\mu \mathrm{mol} \cdot \mathrm{mol}^{-1} \mathrm{CO}_{2}$, at 90, 180, and $270 \mu \mathrm{mol} \cdot \mathrm{m}^{-2} \cdot \mathrm{s}^{-1} P P F D$, respectively, but increased only $20 \%, 16 \%$, and $5 \%$, respectively, from 400 to $600 \mu \mathrm{mol} \cdot \mathrm{mol}^{-1} \mathrm{CO}_{2}$, and there was almost no change from 600 to $800 \mu \mathrm{mol} \cdot \mathrm{mol}^{-1} \mathrm{CO}_{2}$. Differences in $\mathrm{P}_{\max }$ at different $\mathrm{CO}_{2}$ concentrations were significant $(P<0.05)$, with the exception of calibrachoa grown at $180 \mu \mathrm{mol} \cdot \mathrm{m}^{-2} \cdot \mathrm{s}^{-1} P P F D$ (Table 1). Most common was a lower value of $\mathrm{P}_{\max }$ at 200 $\mu \mathrm{mol} \cdot \mathrm{mol}^{-1} \mathrm{CO}_{2}$ compared with higher $\mathrm{CO}_{2}$ concentrations.

Across all $\mathrm{GE}$ and $\mathrm{CO}_{2}$ concentrations, $\alpha$ ranged from 0.0425 to $0.0694 \mu \mathrm{mol} \mathrm{CO}_{2}$ per micromole photons in verbena, 0.0392 to 0.0729 in petunia, and 0.0361 to 0.0727 in calibrachoa (Table 1). These values fell within the range typically observed for $\alpha(0.040$ to 0.075$)$ at ambient $\left(350 \mu \mathrm{mol} \cdot \mathrm{mol}^{-1}\right) \mathrm{CO}_{2}$ (Cannell and Thornley, 1998), although they were slightly below this general range at sub-ambient $\mathrm{CO}_{2}$ concentrations. Where significant, $\alpha$ at 200 $\mu \mathrm{mol} \cdot \mathrm{mol}^{-1} \mathrm{CO}_{2}$ was lower than at 600 and $800 \mu \mathrm{mol} \cdot \mathrm{mol}^{-1}$ $\mathrm{CO}_{2}$, and similar to or lower than at $400 \mu \mathrm{mol} \cdot \mathrm{mol}^{-1} \mathrm{CO}_{2}$.

Dark respiration ranged from 0.53 to $1.28 \mu \mathrm{mol} \cdot \mathrm{m}^{-2} \cdot \mathrm{s}^{-1} \mathrm{CO}_{2}$ in verbena, 0.45 to $1.34 \mu \mathrm{mol} \cdot \mathrm{m}^{-2} \cdot \mathrm{s}^{-1} \mathrm{CO}_{2}$ in petunia, and 0.29 to $0.98 \mu \mathrm{mol} \cdot \mathrm{m}^{-2} \cdot \mathrm{s}^{-1} \mathrm{CO}_{2}$ in calibrachoa (Table 1 ). In all three species, there was a trend for $\mathrm{R}_{\mathrm{d}}$ to generally decrease with increasing $\mathrm{CO}_{2}$ concentration; however, significant differences were not detected within any of the combinations of species and GEs.

The LCP ranged from 8.9 to $25.6 \mu \mathrm{mol} \cdot \mathrm{m}^{-2} \cdot \mathrm{s}^{-1} P P F D$ in verbena, 7.3 to $30.7 \mu \mathrm{mol} \cdot \mathrm{m}^{-2} \cdot \mathrm{s}^{-1} P P F D$ in petunia, and 4.9 to $28.9 \mu \mathrm{mol} \cdot \mathrm{m}^{-2} \cdot \mathrm{s}^{-1} \mathrm{PPFD}$ in calibrachoa (Table 1). The LCP generally decreased with increasing $\mathrm{CO}_{2}$ concentration, and differences were significant within all species and GE combinations, except for verbena grown at $180 \mu \mathrm{mol} \cdot \mathrm{m}^{-2} \cdot \mathrm{s}^{-1} P P F D$.

The calculated LSP, while not compared statistically across light intensities (due to lack of chamber replication), was higher in verbena and petunia acclimated to 180 and $270 \mu \mathrm{mol} \cdot \mathrm{m}^{-2} \cdot \mathrm{s}^{-1}$ $P P F D$, compared with those acclimated at $90 \mu \mathrm{mol} \cdot \mathrm{m}^{-2} \cdot \mathrm{s}^{-1}$ $P P F D$ (Table 1). The LSP generally increased as $\mathrm{CO}_{2}$ concentration increased from 200 to $600 \mu \mathrm{mol} \cdot \mathrm{mol}^{-1}$, then decreased slightly from 600 to $800 \mu \mathrm{mol} \cdot \mathrm{mol}^{-1}$.

EXPT. 2. Pepper and sunflower showed dramatically increased $\mathrm{P}_{\mathrm{n}}$ as growth chamber temperature increased, especially from 14 to $21^{\circ} \mathrm{C}$ (Fig. 2). In pepper, $\mathrm{P}_{\max }$ at the four $\mathrm{CO}_{2}$ concentrations evaluated ranged from 1.0 to $4.2 \mu \mathrm{mol} \cdot \mathrm{m}^{-2} \cdot \mathrm{s}^{-1} \mathrm{CO}_{2}, 7.6$ to 19.6 $\mu \mathrm{mol} \cdot \mathrm{m}^{-2} \cdot \mathrm{s}^{-1} \mathrm{CO}_{2}$, and 8.3 to $26.7 \mu \mathrm{mol} \cdot \mathrm{m}^{-2} \cdot \mathrm{s}^{-1} \mathrm{CO}_{2}$ at 14,21 , and $28^{\circ} \mathrm{C}$, respectively (Table 2 ). In sunflower, $\mathrm{P}_{\max }$ ranged from 8.2 to $30.0 \mu \mathrm{mol} \cdot \mathrm{m}^{-2} \cdot \mathrm{s}^{-1} \mathrm{CO}_{2}, 17.7$ to $53.1 \mu \mathrm{mol} \cdot \mathrm{m}^{-2} \cdot \mathrm{s}^{-1} \mathrm{CO}_{2}$, and 18.2 to $65.1 \mu \mathrm{mol} \cdot \mathrm{m}^{-2} \cdot \mathrm{s}^{-1} \mathrm{CO}_{2}$ at 14,21 , and $28^{\circ} \mathrm{C}$ respectively (Table 2). However, in geranium, higher rates of $\mathrm{P}_{\mathrm{n}}$ were attained at $21^{\circ} \mathrm{C}$ than at 14 or $28^{\circ} \mathrm{C}$. Lower rates of $\mathrm{P}_{n}$ at $28^{\circ} \mathrm{C}$ were likely due to high temperature-induced leaf yellowing and photobleaching (loss of chlorophyll). In all three species, $\mathrm{P}_{\max }$ increased with increasing $\mathrm{CO}_{2}$ concentration, although the magnitude of increase was generally less at higher $\mathrm{CO}_{2}$ concentrations. For example, in sunflower at $21^{\circ} \mathrm{C}, \mathrm{P}_{\max }$ increased $150 \%$ from 200 to $400 \mu \mathrm{mol} \cdot \mathrm{mol}^{-1} \mathrm{CO}_{2}, 20 \%$ from 400 to $600 \mu \mathrm{mol} \cdot \mathrm{mol}^{-1}$, and by less than $1 \%$ from 600 to $800 \mu \mathrm{mol} \cdot \mathrm{mol}^{-1}$. In most instances, $\mathrm{P}_{\max }$ at $200 \mu \mathrm{mol} \cdot \mathrm{mol}^{-1} \mathrm{CO}_{2}$ was significantly lower $(P<0.05)$ than the other three $\mathrm{CO}_{2}$ concentrations, and $\mathrm{P}_{\max }$ at 600 and $800 \mu \mathrm{mol} \cdot \mathrm{mol}^{-1} \mathrm{CO}_{2}$ were similar (Table 2).

Values of $\alpha$ ranged from 0.0137 to $0.0765 \mu \mathrm{mol} \mathrm{CO}_{2}$ per micromole photons in pepper, 0.0398 to 0.0693 in sunflower, and 0.0213 


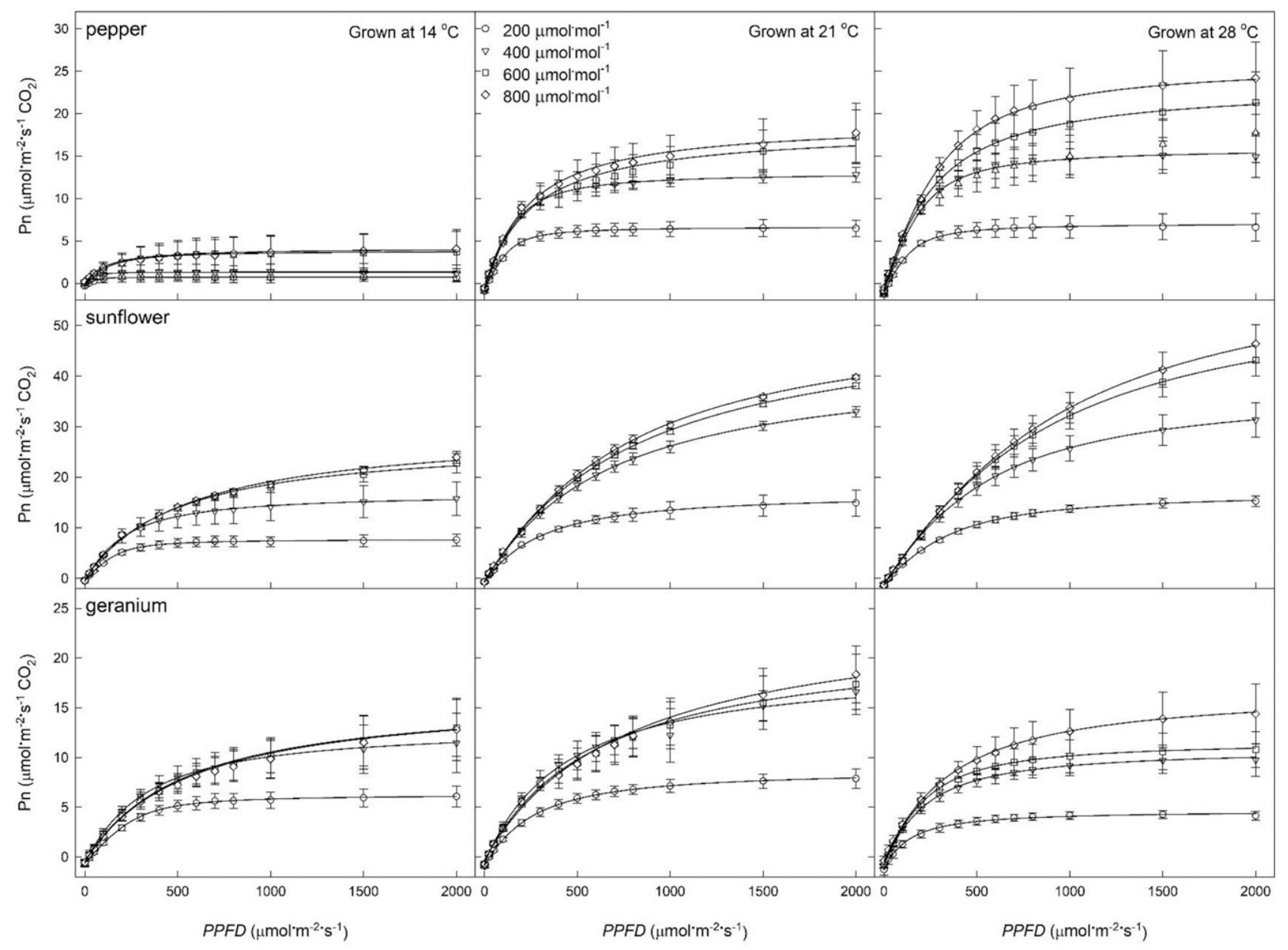

Fig. 2. Net photosynthesis $\left(\mathrm{P}_{\mathrm{n}}\right)$ of pepper 'California Wonder', sunflower 'Pacino Gold', and geranium 'Maverick Red' (top, middle, and bottom rows, respectively) grown at air temperatures of 14,21 , and $28^{\circ} \mathrm{C}$ (left, center, and right columns, respectively). Plants were measured at 14 photosynthetic photon flux densities $(P P F D s)$ and four $\mathrm{CO}_{2}$ concentrations. Points plotted are the means $( \pm \mathrm{SD})$ of each treatment $(\mathrm{n}=5)$ at each $P P F D$. Lines are the modeled values using the equation $P_{n}=1 / 2 \theta\left\{\alpha I+P_{\max }-\left[\left(\alpha I+P_{\max }\right)^{2}-4 \alpha \theta P_{\max } \cdot I\right]^{0.5}\right\}-R_{d}$, with the parameter values listed in Table 2.

to 0.0460 in geranium (Table 2). Significant differences occurred in eight of the nine treatments (species $\times$ GE combinations), but the relationship was inconsistent. In general, $\alpha$ was lowest at 200 $\mu \mathrm{mol} \cdot \mathrm{mol}^{-1} \mathrm{CO}_{2}$. While not statistically compared across temperatures due to lack of replication of GE, $\alpha$ tended to be lower at $14{ }^{\circ} \mathrm{C}$ compared with 21 or $28{ }^{\circ} \mathrm{C}$ but only at $200 \mu \mathrm{mol} \cdot \mathrm{mol}^{-1} \mathrm{CO}_{2}$.

Dark respiration ranged from 0.01 to $1.17 \mu \mathrm{mol} \cdot \mathrm{m}^{-2} \cdot \mathrm{s}^{-1} \mathrm{CO}_{2}$ in pepper, 0.18 to $1.36 \mu \mathrm{mol} \cdot \mathrm{m}^{-2} \cdot \mathrm{s}^{-1} \mathrm{CO}_{2}$ in sunflower, and 0.31 to $1.19 \mu \mathrm{mol} \cdot \mathrm{m}^{-2} \cdot \mathrm{s}^{-1} \mathrm{CO}_{2}$ in geranium (Table 2). Although statistical differences were detected in only four of the nine treatment combinations, $R_{d}$ tended to increase with temperature and typically decreased as $\mathrm{CO}_{2}$ concentration increased.

The LCP ranged from 0.4 to $29.3 \mu \mathrm{mol} \cdot \mathrm{m}^{-2} \cdot \mathrm{s}^{-1} P P F D$ in pepper, 3.2 to $26.4 \mu \mathrm{mol} \cdot \mathrm{m}^{-2} \cdot \mathrm{s}^{-1} P P F D$ in sunflower, and 8.7 to 38.8 $\mu \mathrm{mol} \cdot \mathrm{m}^{-2} \cdot \mathrm{s}^{-1} P P F D$ in geranium (Table 2). The LCP generally decreased with increasing $\mathrm{CO}_{2}$ concentration. For example, in pepper at $28^{\circ} \mathrm{C}$, the LCP was $26.3,15.5,12.9$, and $9.7 \mu \mathrm{mol} \cdot \mathrm{m}^{-2} \cdot \mathrm{s}^{-1}$ PPFD at 200, 400, 600, and $800 \mu \mathrm{mol} \cdot \mathrm{mol}^{-1} \mathrm{CO}_{2}$, respectively.

The LSP ranged from 143 to $>2000 \mu \mathrm{mol} \cdot \mathrm{m}^{-2} \cdot \mathrm{s}^{-1}$ in pepper, 533 to $>2000 \mu \mathrm{mol} \cdot \mathrm{m}^{-2} \cdot \mathrm{s}^{-1}$ in sunflower, and 803 to $>2000 \mu \mathrm{mol} \cdot \mathrm{m}^{-2} \cdot \mathrm{s}^{-1}$ in geranium (Table 2). These values were calculated as the light intensity corresponding to $90 \%$ of net $\mathrm{P}_{\max }$. Based on our fitted equation of $\mathrm{P}_{\mathrm{n}}$ measured at light intensities of $\leq 2000 \mu \mathrm{mol} \cdot \mathrm{m}^{-2} \cdot \mathrm{s}^{-1} P P F D$, some of the calculated values exceeded our upper bound. These extrapolated values may not be biologically realistic and, therefore, are reported in Table 2 as $>2000 \mu \mathrm{mol} \cdot \mathrm{m}^{-2} \cdot \mathrm{s}^{-1} P P F D$. In general, the LSP was higher at 21 and $28^{\circ} \mathrm{C}$, compared with $14^{\circ} \mathrm{C}$. The LSP generally increased with increasing $\mathrm{CO}_{2}$ concentration from 200 to 600 , then either increased or decreased from 600 to 800 .

Fitting to THE CANNELl AND THORNLEY MODEL. Values of $\alpha_{\text {ref }}, \beta, \theta, c_{T, \alpha}, P_{\text {max,ref }}, K_{C a, P \max }, T_{\max , P \max , 400}, T_{\max , P \max , 800}, T_{0}$, and $R_{d}$ were determined by the fitting procedure for geranium, pepper, and sunflower (Table 3). Rates of $\mathrm{P}_{\max }$ increased with $\mathrm{CO}_{2}$ concentration, although the magnitude of increase was incrementally smaller with increasing $\mathrm{CO}_{2}$ concentration. This behavior is captured by Eq. [6], in which $f_{C a, P \max }$, the factor representing the influence of $\mathrm{CO}_{2}$ concentration on $\mathrm{P}_{\max }$, increased asymptotically due to increasing $\mathrm{CO}_{2}$ concentration. The measured behavior of $\mathrm{P}_{\max }$ was also well-represented by Eq. [7], in which $\mathrm{P}_{\max }$ increased up to a temperature optimum, above which it decreased. The fitted temperature optima fell between 20.9 and $26.5^{\circ} \mathrm{C}$ (Table 3 ).

Cannell and Thornley (1998) predicted $\alpha$ to decrease as temperature increased above $15^{\circ} \mathrm{C}$ (Eq. [4], assuming a positive value for $\mathrm{c}_{\mathrm{T}}$ ), based on a review of multiple studies. However, in 
Table 2. Nonrectangular hyperbola equation parameters [maximum photosynthetic rate $\left(\mathrm{P}_{\max }\right)$, quantum yield $(\alpha)$, $\theta$, and dark respiration $\left(\mathrm{R}_{\mathrm{d}}\right)$ ] for photosynthetic light response curves of pepper, sunflower, and geranium grown at three air temperatures $\left(14,21\right.$, and $\left.28^{\circ} \mathrm{C}\right)$ and measured at four $\mathrm{CO}_{2}$ concentrations $\left(200,400,600\right.$, and $\left.800 \mu \mathrm{mol} \cdot \mathrm{mol}^{-1}\right)$. The light compensation point [LCP; photosynthetic photon flux densities $(P P F D)$ at which net photosynthesis $\left(\mathrm{P}_{\mathrm{n}}\right)=0$ ] and light saturation point (LSP; $P P F D$ corresponding to $90 \%$ net $\mathrm{P}_{\text {max }}$ ) were calculated from the nonrectangular hyperbola, $\mathrm{P}_{\mathrm{n}}=1 / 2 \theta\left\{\alpha \mathrm{I}+\mathrm{P}_{\max }-\left[\left(\alpha \mathrm{I}+\mathrm{P}_{\max }\right)^{2}-4 \alpha \theta \mathrm{P}_{\max } I\right]^{0.5}\right\}-\mathrm{R}_{\mathrm{d}}$. All fitted parameters and calculated values are mean \pm SD $(n=5)$.

\begin{tabular}{|c|c|c|c|c|c|c|c|}
\hline \multirow[b]{2}{*}{$\mathrm{GE} / \mathrm{CO}_{2}{ }^{\mathrm{z}}$} & $\begin{array}{c}\mathrm{P}_{\max } \\
\left(\mu \mathrm{mol} \cdot \mathrm{m}^{-2} \cdot \mathrm{s}^{-1}\right. \\
\left.\mathrm{CO}_{2}\right) \\
\end{array}$ & $\begin{array}{c}\alpha\left[\mathrm{CO}_{2}\right. \\
\left(\mu \mathrm{mol} \cdot \mu \mathrm{mol}^{-1}\right. \\
\text { photons })]\end{array}$ & $\theta$ & $\begin{array}{c}\mathrm{R}_{\mathrm{d}} \\
\left(\mu \mathrm{mol} \cdot \mathrm{m}^{-2} \cdot \mathrm{s}^{-1}\right. \\
\left.\mathrm{CO}_{2}\right) \\
\end{array}$ & $\begin{array}{c}\mathrm{LSP} \\
\left(\mu \mathrm{mol} \cdot \mathrm{m}^{-2} \cdot \mathrm{s}^{-1}\right) \\
\end{array}$ & $\begin{array}{c}\mathrm{LCP} \\
\left(\mu \mathrm{mol} \cdot \mathrm{m}^{-2} \cdot \mathrm{s}^{-1}\right) \\
\end{array}$ & \multirow{2}{*}{$\begin{array}{c}\text { Pseudo } R^{2} \\
\left(1-\mathrm{SS}_{\text {error }} / \mathrm{SS}_{\text {total }}\right)^{\mathrm{y}}\end{array}$} \\
\hline & \multicolumn{6}{|c|}{$($ mean $\pm \mathrm{SD})$} & \\
\hline \multicolumn{8}{|c|}{ Pepper 'California Wonder' } \\
\hline $14 / 200$ & $1.0 \pm 0.6$ & $0.0137 \pm 0.0039 \mathrm{~b}^{\mathrm{x}}$ & $0.880 \pm 0.068 \mathrm{a}$ & $0.31 \pm 0.08 \mathrm{a}$ & $214 \pm 140 \mathrm{~b}$ & $29.3 \pm 19.0 \mathrm{a}$ & 0.322 \\
\hline $14 / 400$ & $1.5 \pm 1.0$ & $0.0215 \pm 0.0085 \mathrm{~b}$ & $0.832 \pm 0.184 \mathrm{a}$ & $0.13 \pm 0.11 \mathrm{~b}$ & $143 \pm 78 b$ & $5.2 \pm 4.9 \mathrm{~b}$ & 0.270 \\
\hline $14 / 600$ & $3.9 \pm 2.5$ & $0.0239 \pm 0.0109 \mathrm{ab}$ & $0.527 \pm 0.358 \mathrm{a}$ & $0.04 \pm 0.08 \mathrm{~b}$ & $700 \pm 374 \mathrm{ab}$ & $1.0 \pm 2.3 \mathrm{~b}$ & 0.401 \\
\hline $14 / 800$ & $4.2 \pm 2.5$ & $0.0361 \pm 0.0066 \mathrm{a}$ & $0.001 \pm 0.000 \mathrm{~b}$ & $0.01 \pm 0.03 \mathrm{~b}$ & $1064 \pm 594 \mathrm{ab}$ & $0.4 \pm 0.8 \mathrm{~b}$ & 0.418 \\
\hline ANOVA & 0.033 & 0.003 & $<0.001$ & $<0.001$ & 0.003 & $<0.001$ & \\
\hline $\operatorname{HSD}^{\mathrm{x}}$ & - & 0.0143 & 0.369 & 0.15 & 652 & 17.9 & \\
\hline $21 / 200$ & $7.6 \pm 1.1 \mathrm{c}$ & $0.0475 \pm 0.0036 \mathrm{~b}$ & $0.799 \pm 0.058 \mathrm{a}$ & $0.87 \pm 0.21$ & $449 \pm 121$ & $18.9 \pm 4.3 \mathrm{a}$ & 0.948 \\
\hline $21 / 400$ & $14.0 \pm 0.9 \mathrm{~b}$ & $0.0694 \pm 0.0037 \mathrm{a}$ & $0.689 \pm 0.087 \mathrm{a}$ & $0.88 \pm 0.17$ & $726 \pm 106$ & $13.1 \pm 2.8 \mathrm{ab}$ & 0.988 \\
\hline $21 / 600$ & $18.8 \pm 3.6 \mathrm{ab}$ & $0.0732 \pm 0.0107 \mathrm{a}$ & $0.028 \pm 0.061 \mathrm{~b}$ & $0.51 \pm 0.42$ & $>2000^{\mathrm{w}}$ & $6.8 \pm 5.5 \mathrm{~b}$ & 0.913 \\
\hline $21 / 800$ & $19.6 \pm 4.4 \mathrm{a}$ & $0.0762 \pm 0.0094 \mathrm{a}$ & $0.165 \pm 0.225 b$ & $0.54 \pm 0.40$ & $>2000$ & $7.1 \pm 5.3 b$ & 0.914 \\
\hline ANOVA & $<0.001$ & $<0.001$ & $<0.001$ & 0.155 & - & 0.002 & \\
\hline HSD & 5.3 & 0.0137 & 0.232 & - & - & 8.3 & \\
\hline $28 / 200$ & $8.3 \pm 2.1 \mathrm{c}$ & $0.0459 \pm 0.0081 \mathrm{~b}$ & $0.752 \pm 0.226 \mathrm{a}$ & $1.17 \pm 0.36$ & $610 \pm 440$ & $26.3 \pm 5.1 \mathrm{a}$ & 0.909 \\
\hline $28 / 400$ & $17.0 \pm 2.8 \mathrm{~b}$ & $0.0653 \pm 0.0074 \mathrm{a}$ & $0.732 \pm 0.146 \mathrm{a}$ & $1.01 \pm 0.37$ & $840 \pm 318$ & $15.5 \pm 4.7 b$ & 0.955 \\
\hline $28 / 600$ & $24.4 \pm 4.6 \mathrm{a}$ & $0.0765 \pm 0.0026 \mathrm{a}$ & $0.360 \pm 0.145 \mathrm{~b}$ & $0.96 \pm 0.36$ & $>2000$ & $12.9 \pm 5.0 \mathrm{~b}$ & 0.938 \\
\hline $28 / 800$ & $26.7 \pm 5.3 \mathrm{a}$ & $0.0685 \pm 0.0055 \mathrm{a}$ & $0.635 \pm 0.129 \mathrm{ab}$ & $0.66 \pm 0.35$ & $1503 \pm 352$ & $9.7 \pm 5.0 \mathrm{~b}$ & 0.936 \\
\hline ANOVA & $<0.001$ & $<0.001$ & 0.006 & 0.193 & - & $<0.001$ & \\
\hline HSD & 7.1 & 0.0113 & 0.300 & - & - & 8.9 & \\
\hline \multicolumn{8}{|c|}{ Sunflower 'Pacino Gold' } \\
\hline $14 / 200$ & $8.2 \pm 1.3 \mathrm{c}$ & $0.0398 \pm 0.0024 \mathrm{c}$ & $0.807 \pm 0.048 \mathrm{a}$ & $0.45 \pm 0.05 \mathrm{ab}$ & $533 \pm 125$ & $11.5 \pm 0.9 \mathrm{a}$ & 0.936 \\
\hline $14 / 400$ & $17.5 \pm 4.0 \mathrm{~b}$ & $0.0693 \pm 0.0100 \mathrm{a}$ & $0.392 \pm 0.298 b$ & $0.61 \pm 0.11 \mathrm{a}$ & $1528 \pm 767$ & $9.2 \pm 1.7 \mathrm{ab}$ & 0.884 \\
\hline $14 / 600$ & $27.9 \pm 3.3 \mathrm{a}$ & $0.0574 \pm 0.0058 \mathrm{ab}$ & $0.040 \pm 0.088 \mathrm{c}$ & $0.30 \pm 0.24 b$ & $>2000$ & $5.1 \pm 4.1 b c$ & 0.987 \\
\hline $14 / 800$ & $30.0 \pm 2.1 \mathrm{a}$ & $0.0544 \pm 0.0065 \mathrm{~b}$ & $0.001 \pm 0.000 \mathrm{c}$ & $0.18 \pm 0.18 b$ & $>2000$ & $3.2 \pm 2.9 \mathrm{c}$ & 0.994 \\
\hline ANOVA & $<0.001$ & $<0.001$ & $<0.001$ & 0.004 & - & $<0.001$ & \\
\hline HSD & 5.2 & 0.0124 & 0.284 & 0.30 & - & 4.9 & \\
\hline $21 / 200$ & $17.7 \pm 3.7 \mathrm{c}$ & $0.0495 \pm 0.0043 b$ & $0.376 \pm 0.297$ & $0.65 \pm 0.06$ & $>2000$ & $13.5 \pm 1.9$ & 0.961 \\
\hline $21 / 400$ & $44.2 \pm 2.2 \mathrm{~b}$ & $0.0626 \pm 0.0060 \mathrm{a}$ & $0.152 \pm 0.109$ & $0.75 \pm 0.22$ & $>2000$ & $12.2 \pm 3.5$ & 0.994 \\
\hline $21 / 600$ & $52.8 \pm 3.1 \mathrm{a}$ & $0.0609 \pm 0.0032 \mathrm{a}$ & $0.208 \pm 0.149$ & $0.59 \pm 0.16$ & $>2000$ & $9.9 \pm 3.0$ & 0.998 \\
\hline $21 / 800$ & $53.1 \pm 2.2 \mathrm{a}$ & $0.0593 \pm 0.0028 \mathrm{a}$ & $0.370 \pm 0.076$ & $0.54 \pm 0.24$ & $>2000$ & $9.3 \pm 4.5$ & 0.998 \\
\hline ANOVA & $<0.001$ & $<0.001$ & 0.153 & 0.341 & - & 0.206 & \\
\hline HSD & 5.2 & 0.0077 & - & - & - & - & \\
\hline $28 / 200$ & $18.2 \pm 1.5 \mathrm{c}$ & $0.0402 \pm 0.0034 \mathrm{~b}$ & $0.598 \pm 0.058$ & $1.04 \pm 0.35$ & $1996 \pm 316$ & $26.4 \pm 7.7$ & 0.990 \\
\hline $28 / 400$ & $38.9 \pm 4.6 b$ & $0.0562 \pm 0.0043 \mathrm{a}$ & $0.518 \pm 0.061$ & $1.11 \pm 0.26$ & $>2000$ & $20.3 \pm 5.5$ & 0.976 \\
\hline $28 / 600$ & $59.5 \pm 2.9 \mathrm{a}$ & $0.0564 \pm 0.0038 \mathrm{a}$ & $0.463 \pm 0.077$ & $1.18 \pm 0.35$ & $>2000$ & $21.2 \pm 6.3$ & 0.987 \\
\hline $28 / 800$ & $65.1 \pm 3.2 \mathrm{a}$ & $0.0557 \pm 0.0036 \mathrm{a}$ & $0.500 \pm 0.098$ & $1.36 \pm 0.30$ & $>2000$ & $24.8 \pm 6.2$ & 0.984 \\
\hline ANOVA & $<0.001$ & $<0.001$ & 0.068 & 0.468 & - & 0.421 & \\
\hline HSD & 5.9 & 0.0068 & - & - & - & - & \\
\hline \multicolumn{8}{|c|}{ Geranium 'Maverick Red' } \\
\hline $14 / 200$ & $6.9 \pm 1.3 \mathrm{~b}$ & $0.0213 \pm 0.0028 \mathrm{~b}$ & $0.829 \pm 0.049 \mathrm{a}$ & $0.63 \pm 0.29$ & $803 \pm 206$ & $29.4 \pm 11.9$ & 0.949 \\
\hline $14 / 400$ & $13.8 \pm 4.0 \mathrm{ab}$ & $0.0376 \pm 0.0068 \mathrm{a}$ & $0.288 \pm 0.341 \mathrm{~b}$ & $0.64 \pm 0.22$ & $>2000$ & $18.6 \pm 8.4$ & 0.903 \\
\hline $14 / 600$ & $16.7 \pm 4.9 \mathrm{a}$ & $0.0303 \pm 0.0037 \mathrm{a}$ & $0.102 \pm 0.226 \mathrm{~b}$ & $0.46 \pm 0.29$ & $>2000$ & $15.6 \pm 9.4$ & 0.919 \\
\hline $14 / 800$ & $16.8 \pm 5.4 \mathrm{a}$ & $0.0302 \pm 0.0031 \mathrm{a}$ & $0.051 \pm 0.078 \mathrm{~b}$ & $0.51 \pm 0.22$ & $>2000$ & $17.6 \pm 8.0$ & 0.908 \\
\hline
\end{tabular}


Table 2. (Continued)

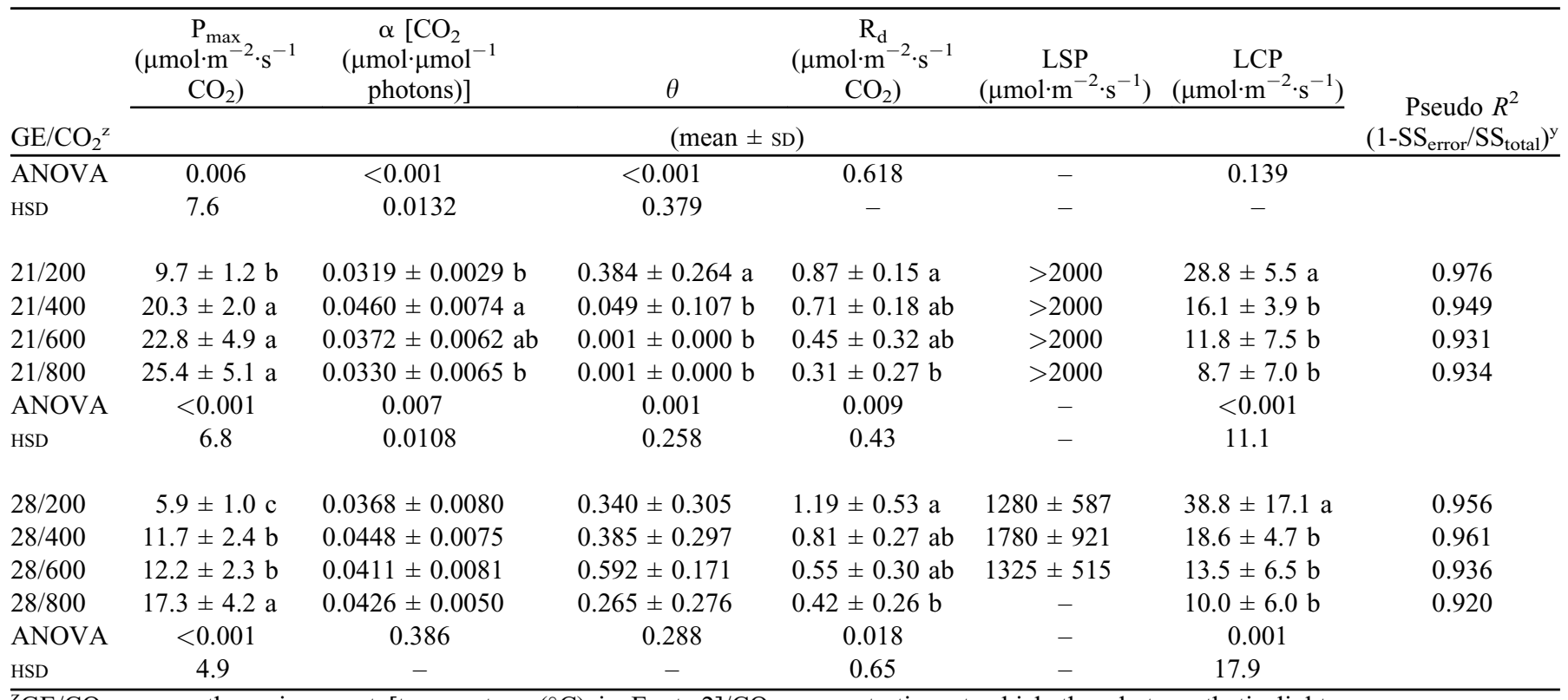

${ }^{\bar{z}} \mathrm{GE} / \mathrm{CO}_{2}=$ growth environment [temperature $\left({ }^{\circ} \mathrm{C}\right)$ in Expt. 2]/CO $\mathrm{CO}_{2}$ concentration at which the photosynthetic light response curve was determined.

${ }^{\mathrm{y}} \mathrm{SS}_{\text {error }} / \mathrm{SS}_{\text {total }}=$ sum of squares (error)/sum of squares (total).

${ }^{\mathrm{x}}$ Tukey's honestly significant difference (HSD). A hyphen for HSD indicates value not reported $(P>0.05)$. Within each species and GE, means followed by different letters are statistically different at $\alpha=0.05$.

${ }^{\mathrm{w}}$ LSP values exceeding the upper bounds evaluated are noted as $>2000 \mu \mathrm{mol} \cdot \mathrm{m}^{-2} \cdot \mathrm{s}^{-1} P P F D$ rather than reporting an extrapolated value; ANOVA not performed for the GE.

the present study, $\alpha$ increased with temperature in pepper and geranium (Table 2). As a result, when fitting our data to the Cannell and Thornley model, the parameter $\mathrm{c}_{\mathrm{T}}$, which represents the impact of temperature on $\alpha$, was negative in geranium and pepper, instead of positive as expected. The Cannell and Thornley model also assumes $\alpha$ increases with $\mathrm{CO}_{2}$ concentration (Eq. [3]). Although our measured values of $\alpha$ at $200 \mu \mathrm{mol} \cdot \mathrm{mol}^{-1}$ were consistently lower than at the higher $\mathrm{CO}_{2}$ concentrations, $\alpha$ did not consistently increase with $\mathrm{CO}_{2}$ concentrations above 400 $\mu \mathrm{mol} \cdot \mathrm{mol}^{-1}$. Mean $\alpha$ for a given species and GE was greater (in value, but not necessarily statistically significant) at a $\mathrm{CO}_{2}$ concentration of $400 \mu \mathrm{mol} \cdot \mathrm{mol}^{-1}$ than at 600 or $800 \mu \mathrm{mol} \cdot \mathrm{mol}^{-1}$ for 15 of 36 species and GE combinations.

The root mean square error (RSME) of the fitted model was compared with the average RMSE from the NRH functions fit to each species. Both values are presented in Table 3. The RMSE of the Cannell and Thornley model for sunflower was 1.57 vs. an average of 1.06 when data from each temperature and $\mathrm{CO}_{2}$ concentration combination were fit individually with an NRH. Likewise, the RMSE of the Cannell and Thornley model for pepper was 1.44 vs. an average of 1.21 when data from each temperature and $\mathrm{CO}_{2}$ concentration combination were fit individually with an $\mathrm{NRH}$, and 1.29 vs. an average of 1.05 , respectively, for geranium.

\section{Discussion}

Previous studies have developed photosynthetic response curves for greenhouse ornamentals to light (e.g., Abreu et al., 2014; Ronquim et al., 2009), $\mathrm{CO}_{2}$ (e.g., Wullschleger, 1993), temperature (e.g., Berry and Björkman, 1980), or some combination (e.g., Boldt et al., 2014; Erwin and Gesick, 2017; Iwakiri and Inayama, 1975), but typically only along lines in the PPFD, $\mathrm{CO}_{2}$ concentration, and temperature variable space; the response to one variable was measured while holding the others constant. For example, data could be used to predict plant responses to a change in light intensity or $\mathrm{CO}_{2}$ concentration where these lines intersect, but not a simultaneous change in both. Increasing (or decreasing) light intensity and $\mathrm{CO}_{2}$ simultaneously can result in interactive effects on $\mathrm{P}_{\mathrm{n}}$, especially in combination with changes to leaf temperature as well. Our study is one of the first to report instantaneous $\mathrm{P}_{\mathrm{n}}$ measurements at multiple combinations of light intensity, $\mathrm{CO}_{2}$ concentration, and leaf temperature in greenhouse ornamentals, which can be used to model the interactive effects on $\mathrm{P}_{\mathrm{n}}$. Stanghellini and Bunce (1993) previously evaluated these same environmental variables (light intensity, $\mathrm{CO}_{2}$, and temperature) on tomato plants grown at ambient $\left(350 \mu \mathrm{mol} \cdot \mathrm{mol}^{-1}\right)$. Although general trends were similar between our results and theirs, our results show significant species-to-species variation. This means that additional studies of this type are warranted to model the responses of more species.

All species evaluated displayed the characteristic photosynthetic light response curve shape regardless of the light intensity or temperature to which they were acclimated or the $\mathrm{CO}_{2}$ concentration during measurement. The NRH parameter values, LCPs, and LSPs (Tables 1 and 2) were generally within expected ranges but also showed substantial differences between results from previous studies measuring the same species. In general, $\mathrm{P}_{\max }$ of petunia, verbena, geranium, and sunflower was higher in the present study compared with Boldt et al. (2014), which may be because of the slightly higher growth and measurement temperature ( 21 to $22^{\circ} \mathrm{C}$, as compared with $20^{\circ} \mathrm{C}$ for PhotoSim), cultivar, GE, and/or plant age. Photosynthetic responses to 
Table 3. Fitted parameters for the Cannell and Thornley model used to model quantum yield $(\alpha)$ and maximum photosynthetic rate $\left(\mathrm{P}_{\max }\right)$ as functions of temperature and $\mathrm{CO}_{2}$ concentration, as well as the root mean squared error (RMSE) of the Cannell and Thornley model using these fitted parameters ( $\left.\mathrm{RMSE}_{\text {Cannell-Thornley }}\right)$ and the average RMSE of each nonrectangular hyperbola, $\mathrm{P}_{\mathrm{n}}=1 / 2 \theta\{\alpha \mathrm{I}+$ $\left.\left.\mathrm{P}_{\max }-\left[\left(\alpha \mathrm{I}+\mathrm{P}_{\max }\right)^{2}-4 \alpha \theta \mathrm{P}_{\max } \mathrm{I}\right]^{0.5}\right]\right\}$ for each species (Avg. RMSE $\left._{\mathrm{NRH}}\right)$.

\begin{tabular}{|c|c|c|c|}
\hline Parameter & Sunflower & Pepper & Geranium \\
\hline$\alpha_{\text {ref }}\left[\mathrm{CO}_{2}\left(\mu \mathrm{mol} \cdot \mu \mathrm{mol}^{-1} \text { photons }\right)\right]^{2}$ & 0.0630 & 0.0672 & 0.0460 \\
\hline$\beta\left(\mu \mathrm{mol} \cdot \mathrm{mol}^{-1}\right)$ & $0.0^{y}$ & 134 & 127 \\
\hline $\mathrm{c}_{\mathrm{T}, \alpha}\left({ }^{\circ} \mathrm{C}^{-1}\right)$ & 0.0104 & -0.0615 & -0.0356 \\
\hline $\mathrm{P}_{\text {max }, \mathrm{ref}}\left(\mu \mathrm{mol} \cdot \mathrm{m}^{-2} \cdot \mathrm{s}^{-1} \mathrm{CO}_{2}\right)$ & 129.9 & 28.7 & 27.7 \\
\hline $\mathrm{K}_{\mathrm{Ca}, \mathrm{Pmax}}\left(\mu \mathrm{mol} \cdot \mathrm{mol}^{-1}\right)$ & 1109 & 498 & 197 \\
\hline $\mathrm{T}_{\max , \operatorname{Pmax}, 400}\left({ }^{\circ} \mathrm{C}\right)$ & 24.4 & 22.4 & 22.5 \\
\hline $\mathrm{T}_{\max , \operatorname{Pmax}, 800}\left({ }^{\circ} \mathrm{C}\right)$ & 26.5 & 24.3 & 20.9 \\
\hline $\mathrm{T}_{0}\left({ }^{\circ} \mathrm{C}\right)$ & 4.19 & 9.73 & -0.49 \\
\hline$\theta$ & 0.355 & 0.386 & 0.001 \\
\hline $\mathrm{R}_{\mathrm{d}}\left(\mu \mathrm{mol} \cdot \mathrm{m}^{-2} \cdot \mathrm{s}^{-1} \mathrm{CO}_{2}\right)$ & 0.978 & 0.79 & 0.559 \\
\hline RMSE $_{\text {Cannell-Thornley }}$ & 1.57 & 1.45 & 1.29 \\
\hline Avg. RMSE $\mathrm{NRH}_{\mathrm{N}}$ & 1.06 & 1.21 & 1.05 \\
\hline
\end{tabular}

${ }^{\mathrm{z}} \alpha_{\text {ref }}=$ the value of $\alpha$ at a reference temperature and $\mathrm{CO}_{2}$ concentration (assumed to be $14{ }^{\circ} \mathrm{C}$ and $400 \mu \mathrm{mol} \cdot \mathrm{mol}^{-1}$ ), $\beta=$ a coefficient affecting the sensitivity of $\alpha$ to $\mathrm{CO}_{2}$ concentration (micromoles per mole), $\mathrm{c}_{\mathrm{T}, \alpha}=\mathrm{a}$ coefficient affecting the sensitivity of $\alpha$ to temperature $\left({ }^{\circ} \mathrm{C}^{-1}\right), \mathrm{P}_{\text {max,ref }}=$ the value of $\mathrm{P}_{\max }$ at a reference temperature and $\mathrm{CO}_{2}$ concentration (assumed to be $20^{\circ} \mathrm{C}$ and $400 \mu \mathrm{mol} \cdot \mathrm{mol}^{-1}$ ), $\mathrm{K}_{\mathrm{Ca} \text {,Pmax }}=\mathrm{a}$ coefficient affecting the sensitivity of $\mathrm{P}_{\max }$ to $\mathrm{CO}_{2}$ concentration (micromoles per mole), $\mathrm{T}_{\max , P \max , 400}=$ the temperature $\left({ }^{\circ} \mathrm{C}\right)$ that maximizes $\mathrm{P}_{\max }$ at $400 \mu \mathrm{mol} \cdot \mathrm{mol}^{-1} \mathrm{CO}_{2}, \mathrm{~T}_{\max , \mathrm{Pmax}, 800}=$ the temperature $\left({ }^{\circ} \mathrm{C}\right)$ that maximizes $\mathrm{P}_{\max }$ at $800 \mu \mathrm{mol} \cdot \mathrm{mol}^{-1} \mathrm{CO}_{2}$, $\mathrm{T}_{0}=$ the lower temperature $\left({ }^{\circ} \mathrm{C}\right)$ at which $\mathrm{P}_{\max }$ is $0, \theta=$ a curvature parameter, and $\mathrm{R}_{\mathrm{d}}=$ dark respiration (micromoles $\mathrm{CO}_{2}$ per square meter per second).

${ }^{\mathrm{y}}$ Value constrained to $\geq 0$ to avoid extreme parameter values.

irradiance in field-grown irrigated pepper 'Quadrato d'Asti' reached an asymptote at $\approx 25 \mu \mathrm{mol} \cdot \mathrm{m}^{-2} \cdot \mathrm{s}^{-1} \mathrm{CO}_{2}$ at a leaf temperature of $25^{\circ} \mathrm{C}$ (Delfine et al., 2001). This is higher than the $\mathrm{P}_{\max }$ of 14.0 and $17.0 \mu \mathrm{mol} \cdot \mathrm{m}^{-2} \cdot \mathrm{s}^{-1} \mathrm{CO}_{2}$ observed in our study at ambient $\mathrm{CO}_{2}\left(400 \mu \mathrm{mol} \cdot \mathrm{mol}^{-1}\right)$ and temperatures of 21 and $28^{\circ} \mathrm{C}$, respectively, and could be attributed to cultivar, higher irradiance in the field relative to a growth chamber, or differences in production systems. We did not find previous reports of light response curves on calibrachoa.

Previously, LCPs of 24 to $52 \mu \mathrm{mol} \cdot \mathrm{m}^{-2} \cdot \mathrm{s}^{-1} P P F D$ in herbaceous ornamentals (Boldt et al., 2014) and 13 to $73 \mu \mathrm{mol} \cdot \mathrm{m}^{-2} \cdot \mathrm{s}^{-1}$ $P P F D$ in leafy greens (Erwin and Gesick, 2017) have been reported at ambient $\mathrm{CO}_{2}\left(400 \mu \mathrm{mol} \cdot \mathrm{mol}^{-1}\right)$, which were higher than the calculated LCP values in our study (4.9 to $30.7 \mu \mathrm{mol} \cdot \mathrm{m}^{-2} \cdot \mathrm{s}^{-1}$ in Expt. 1 and 0.4 to $38.8 \mu \mathrm{mol} \cdot \mathrm{m}^{-2} \cdot \mathrm{s}^{-1}$ in Expt. 2). The lowest LCPs calculated in our study were typically at 600 and 800 $\mu \mathrm{mol} \cdot \mathrm{mol}^{-1} \mathrm{CO}_{2}$, which may explain why some of our LCPs were lower than those reported for other ornamentals. The LCP, the irradiance intensity at which photosynthetic carbon fixation offsets carbon losses from dark respiration and photorespiration, generally decreases as acclimated light intensity decreases or $\mathrm{CO}_{2}$ concentration increases. This corresponds with what we observed. The reduction in $\mathrm{LCP}$ as $\mathrm{CO}_{2}$ increased indicates that growers may be able to partially offset the impacts of very low ambient irradiance intensities early in the growing season by increasing $\mathrm{CO}_{2}$; however, this strategy may be more effective at the vegetative stage, when canopy shading is minimal (low leaf area index) and plant biomass is often a primary quality metric. At the reproductive stage, however, light intensity is an important contributor not just to biomass accumulation but flower initiation and time to flower as well, and may not be able to be offset by $\mathrm{CO}_{2}$ supplementation.

In general, the LSP increased with increasing $\mathrm{CO}_{2}$ concentration. At elevated $\mathrm{CO}_{2}$ concentration $\left(>400 \mu \mathrm{mol} \cdot \mathrm{mol}^{-1}\right)$, some LSP values were quite high, above $2000 \mu \mathrm{mol} \cdot \mathrm{m}^{-2} \cdot \mathrm{s}^{-1} P P F D$, and involved extrapolating outside the tested domain. Therefore, they are only reported as $>2000 \mu \mathrm{mol} \cdot \mathrm{m}^{-2} \cdot \mathrm{s}^{-1}$ in Tables 1 and 2 . In addition, values $>2000 \mu \mathrm{mol} \cdot \mathrm{m}^{-2} \cdot \mathrm{s}^{-1}$ are unlikely in greenhouses or other controlled environments. The lower values of LSP calculated likely have more practical meaning to growers. For instance, in pepper at $14{ }^{\circ} \mathrm{C}$ and $200 \mu \mathrm{mol} \cdot \mathrm{mol}^{-1} \mathrm{CO}_{2}$, the measured LSP was only $214 \mu \mathrm{mol} \cdot \mathrm{m}^{-2} \cdot \mathrm{s}^{-1} \mathrm{PPFD}$. Such information can be useful for deciding on light, temperature, and $\mathrm{CO}_{2}$ set points during winter, especially at higher latitudes. At $14{ }^{\circ} \mathrm{C}$ and $200 \mu \mathrm{mol} \cdot \mathrm{mol}^{-1} \mathrm{CO}_{2}$, adding additional light above the LSP would likely be an unnecessary expense and not contribute to increased growth, unless the $\mathrm{CO}_{2}$ concentration was also concurrently increased or unless the crop canopy was quite dense, in which case the additional light would penetrate deeper into the canopy. Rather, increasing the ambient temperature would be more beneficial. It is worth noting that there are limitations in extrapolating individual leaf responses to whole-plant or canopy measures of $\mathrm{P}_{\mathrm{n}}$ (Erwin and Gesick, 2017), e.g., plant age or leaf canopy denseness and depth (and subsequently, the degree of leaf shading). However, modeling these instantaneous measurements, along with knowledge of the crop age, growth stage, and the current environmental conditions they are acclimated to, can provide a starting framework to adjust temperature, irradiance, and $\mathrm{CO}_{2}$ to optimize growth and yield or maximize biomass production per cost of inputs.

In Expt. 1, we wanted to see if the light intensity of the growth chamber in which plants were grown before measurement impacted photosynthetic response. The increased $\mathrm{P}_{\max }$ observed in verbena and petunia acclimated to higher light intensities agreed with previous findings that the higher the light intensity during growth, the higher the photosynthetic capacity of the leaf will be (Bunce and Heichel, 1986). However, this was not the case with calibrachoa. It is possible that the inconsistent results between GE were due to selection bias. As noted in the methods, many of the recently mature calibrachoa leaves of plants grown at 180 and $270 \mu \mathrm{mol} \cdot \mathrm{m}^{-2} \cdot \mathrm{s}^{-1} P P F D$ were too small to cover even the smaller $\left(2 \mathrm{~cm}^{2}\right)$ cuvette used to measure $\mathrm{P}_{\mathrm{n}}$ of calibrachoa and petunia; therefore, larger, older leaves (that unfolded and matured in the lighting environment) and did fully cover the small cuvette were measured in those instances. Studies (e.g., Iwakiri and Inayama, 1975) have shown that older leaves can have lower rates of $\mathrm{P}_{n}$.

We observed that all three species (calibrachoa, petunia, and verbena) had larger, but fewer leaves at the lowest light intensity and smaller, but more tightly packed leaves at the higher light intensities. These differences can be attributed to morphological responses to light intensity. First, leaves acclimated to lower light intensities tend to be larger and thinner, compared with leaves acclimated to higher light intensities, as a way to maximize light capture (Poorter et al., 2019). Second, internode length often increases as light intensity decreases, leading to a less "tightly packed" appearance (Garcia and Lopez, 2020; Poorter et al., 2019). 
In Expt. 2, $\mathrm{P}_{\max }$ increased with increasing GE temperature in sunflower and pepper, but not in geranium. At $14^{\circ} \mathrm{C}, \mathrm{P}_{\mathrm{n}}$ in pepper was especially low. This shows that adding supplemental $\mathrm{CO}_{2}$ and/or light to pepper (a cold-sensitive plant) would increase $\mathrm{P}_{\mathrm{n}}$ somewhat at $14^{\circ} \mathrm{C}$, but not nearly as much as increasing the temperature. In geranium, $\mathrm{P}_{\max }$ increased from 14 to $21^{\circ} \mathrm{C}$, then declined at $28^{\circ} \mathrm{C}$. Geraniums grown at $28^{\circ} \mathrm{C}$ exhibited photobleaching on leaves that developed after placement in the temperature treatments, a physiological response sometimes observed in geranium in response to high temperature (Owen and Lindberg, 2017). Several measurements on the yellowed leaves resulted in very low rates of $\mathrm{P}_{\mathrm{n}}$. Therefore, older (but greener) leaves were selected for measurement. Also, the lower $\mathrm{P}_{\max }$ in geranium at 28 vs. $21^{\circ} \mathrm{C}$ could also be attributed to its calculated temperature optimum of $22.5^{\circ} \mathrm{C}$ at 400 $\mu \mathrm{mol} \cdot \mathrm{mol}^{-1}$ (Table 3), which is close to the $21^{\circ} \mathrm{C}$ environment. The temperature optimum for plants in Expt. 2 was determined to be between 20.9 and $26.5^{\circ} \mathrm{C}$, based on regression performed for fitting the Cannell and Thornley model. This fits within the temperature optimum range of 11.1 to $30.8^{\circ} \mathrm{C}$ that Boldt et al. (2014) reported for 13 herbaceous ornamentals.

This study may be the first, to our knowledge, that fits NRH parameter values to empirical data to model $\mathrm{P}_{\mathrm{n}}$ as a function of light intensity, $\mathrm{CO}_{2}$ concentration, and leaf temperature, allowing for the estimation of $\mathrm{P}_{\mathrm{n}}$ at any combination of these three variables within the domain tested. The Cannell and Thornley model was a good starting point, although we adjusted the reference values of temperature $\left(14^{\circ} \mathrm{C}\right.$ instead of $\left.15^{\circ} \mathrm{C}\right)$ and $\mathrm{CO}_{2}\left(400 \mu \mathrm{mol} \cdot \mathrm{mol}^{-1}\right.$ instead of $\left.350 \mu \mathrm{mol} \cdot \mathrm{mol}^{-1}\right)$ and included $\mathrm{R}_{\mathrm{d}}$ to better model our scenario, as described in the materials and methods.

Contour plots for sunflower (Fig. 3) show a comparison of $\mathrm{P}_{\mathrm{n}}$ response surfaces interpolated for light intensity and $\mathrm{CO}_{2}$ by the NRH functions (Fig. 3A) and the fitted Cannell and Thornley model (Fig. 3B). The similarities indicate either one could be used as a decision-support tool. However, the NRH surface plots are limited to the three temperatures evaluated, whereas the fitted Cannell and Thornley model could produce a plot at any temperature between 14 and $28^{\circ} \mathrm{C}$. The quality of fit of the Cannell and Thornley model to our experimental data (RMSE of 1.3 to 1.6, depending on species) may be adequate for a number of applications, including decision support for greenhouse climate control. The Cannell and Thornley model fits all the experimental data with a single model, as compared with fitting separate NRHs for each temperature and $\mathrm{CO}_{2}$ concentration, hence the greater RMSE compared with individual NRH models (comparison of RMSE in Table 3). However, the small tradeoff in accuracy may be worthwhile for applications that require predicting $\mathrm{P}_{\mathrm{n}}$ at conditions that do not fall along one of the NRHs, as it would be experimentally impractical to measure and develop $\mathrm{P}_{\mathrm{n}}$ response curves for irradiance and $\mathrm{CO}_{2}$ at each degree or couple of degrees Celsius for multiple species and cultivars.

Interestingly, we observed an increase in $\alpha$ with increased temperature in pepper and geranium, instead of the expected
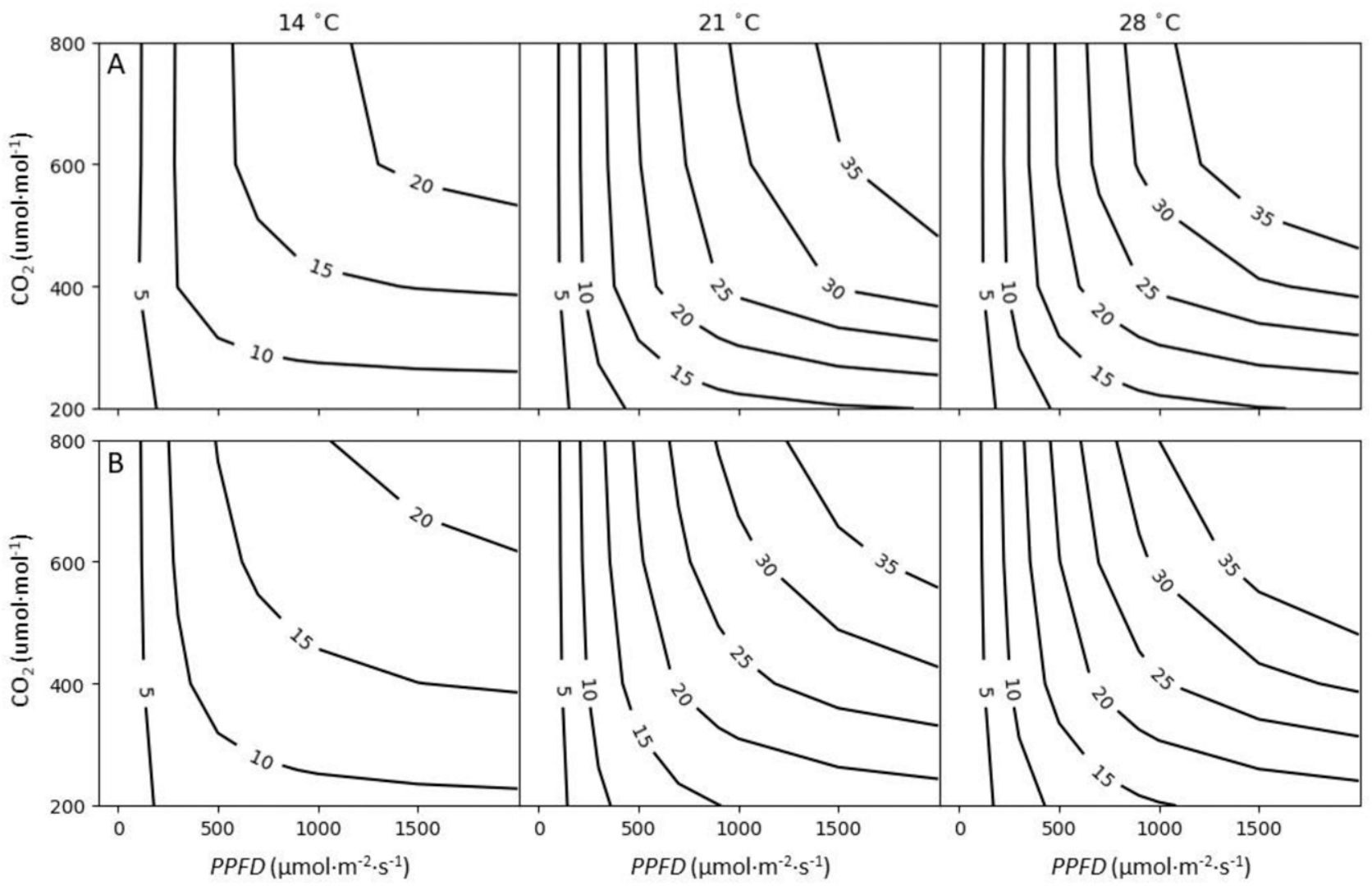

Fig. 3. Comparison of net photosynthesis $\left(\mathrm{P}_{\mathrm{n}}\right)$ of sunflower 'Pacino Gold' modeled by fitting the nonrectangular hyperbola (NRH) equation to data at 14 , 21 , and $28^{\circ} \mathrm{C}$ and each $\mathrm{CO}_{2}$ concentration $(\mathbf{A})$ and by fitting the Cannell and Thornley model to the same data $(\mathbf{B})$. The NRH equation: $\mathrm{P}_{\mathrm{n}}=1 / 2 \theta\left\{\alpha \mathrm{I}+\mathrm{P}_{\max }-\right.$ $\left.\left[\left(\alpha \mathrm{I}+\mathrm{P}_{\max }\right)^{2}-4 \alpha \theta \mathrm{P}_{\max } \mathrm{I}\right]^{0.5}\right\}-\mathrm{R}_{\mathrm{d}}$. The Cannell and Thornley model includes equations for $\alpha$ and $\mathrm{P}_{\max }$ as functions of temperature and $\mathrm{CO}_{2}$ concentration. 
decrease. It is possible that differences in plant development during growth at different temperatures accounts for this. Peri et al. (2005) found that $\alpha$ decreased by $\approx 40 \%$ when leaf nitrogen $(\mathrm{N})$ decreased from $4 \%$ to $2 \%$, which corresponded to a decrease in total chlorophyll, from $\approx 0.6$ to $0.1 \mathrm{~g} \cdot \mathrm{m}^{-2}$. Although we did not measure $\mathrm{N}$ directly, previous research [e.g., review by Sinclair and Horie (1989)] has shown a high correlation between $P_{n}$ per unit leaf area and leaf $\mathrm{N}$ per unit leaf area. As such, it is possible that leaf $\mathrm{N}$ may have been lower at $14^{\circ} \mathrm{C}$ for pepper and geranium, compared with 21 or $28^{\circ} \mathrm{C}$, and this relationship may be responsible for the increased values of $\alpha$ at the higher temperatures. Johnson et al. (2010) built on the Cannell and Thornley model using the results from Peri et al. (2005) by incorporating a factor for protein concentration into the expressions for $\alpha$ and $\mathrm{P}_{\max }$. If leaf $\mathrm{N}$ had been measured, the incorporation of this factor may have improved the fit of the model.

One application of either the NRHs or the fitted Cannell and Thornley model is that they allow growers to assess various combinations of environmental conditions simultaneously, rather than individually. This provides them with the opportunity to see which sets of conditions would result in similar rates of $\mathrm{P}_{\mathrm{n}}$. In Fig. 3, a similar rate of $P_{n}$ could be achieved at any point located on a contour within the plot. For instance, in sunflower grown at $21^{\circ} \mathrm{C}$ (Fig. 3), a $\mathrm{P}_{\mathrm{n}}$ of $20 \mu \mathrm{mol} \cdot \mathrm{m}^{-2} \cdot \mathrm{s}^{-1} \mathrm{CO}_{2}$ is achievable at $\approx 600 \mu \mathrm{mol} \cdot \mathrm{mol}^{-1} \mathrm{CO}_{2}$ and $500 \mu \mathrm{mol} \cdot \mathrm{m}^{-2} \cdot \mathrm{s}^{-1}$ PPFD or at 300 $\mu \mathrm{mol} \cdot \mathrm{mol}^{-1} \mathrm{CO}_{2}$ and $1000 \mu \mathrm{mol} \cdot \mathrm{m}^{-2} \cdot \mathrm{s}^{-1}$ PPFD. At a temperature of $14^{\circ} \mathrm{C}$, much higher levels of light intensity and $\mathrm{CO}_{2}$ concentration would be needed to attain the same $\mathrm{P}_{\mathrm{n}}$. Based on the species being grown, geographic location, time of year, greenhouse light transmission, electricity and $\mathrm{CO}_{2}$ costs, and other associated factors, a grower could use these plots, or an associated decision support tool that incorporates them, to assess which combination of light intensity, $\mathrm{CO}_{2}$ concentration, and temperature is most feasible and economically sound for them to achieve the desired $\mathrm{P}_{\mathrm{n}}$ at a minimum cost. In the northern United States, low ambient irradiance is a limiting factor during winter and early spring greenhouse production. Supplemental lighting, historically from high-pressure sodium lamps but now also including LEDs, can be quite expensive to install and operate. If $\mathrm{CO}_{2}$ supplementation could partially or substantially reduce the need for supplemental lighting, even for a few hours per day when venting is not actively occurring or imminent, without reducing $P_{n}$, cost savings may be realized. This is likely to be more advantageous to growers producing leafy crops, ornamental or edible, or during the vegetative stage of crop growth, where the impact of DLI on flower initiation and development is not critical to crop timing.

In lieu of $\mathrm{CO}_{2}$ supplementation from liquid $\mathrm{CO}_{2}$ or $\mathrm{CO}_{2}$ generators, growers could temporally increase the $\mathrm{CO}_{2}$ concentration of the greenhouse, from sub-ambient to near ambient, by opening vents periodically and using fans to mix the air around the plant canopy to reduce the boundary layer. These strategies would be most feasible when venting is already scheduled for humidity or temperature control; if not, the tradeoff for increasing the $\mathrm{CO}_{2}$ concentration would be the loss of pre-heated air from the greenhouse and higher heating costs. A cost calculator, based on greenhouse location, time of year, and desired crop growth rates would benefit growers.

In addition, plant acclimation to supplemental $\mathrm{CO}_{2}$ should be considered. Although most crops exhibit an overall positive response to $\mathrm{CO}_{2}$ enrichment (Kimball, 1983), they will begin acclimating after a few weeks (McKinney and Craver, 2021; Yelle et al., 1990). Therefore, $\mathrm{CO}_{2}$ supplementation may only be early in the cropping cycle when plants are most responsive. For plants with short crop cycles, such as annual bedding plants, $\mathrm{CO}_{2}$ supplementation may be appropriate throughout production, especially if the greenhouse contains plants at multiple growth stages. Due to acclimation, photosynthetic and growth models developed from instantaneous changes in $\mathrm{P}_{\mathrm{n}}$ to $\mathrm{CO}_{2}$ concentration will likely overestimate plant response as crop duration increases.

In summary, our data are one of the first studies to report instantaneous $\mathrm{P}_{\mathrm{n}}$ measurements for ornamental species at multiple combinations of light intensity, $\mathrm{CO}_{2}$ concentration, leaf temperature, and GE, which can be used to model their interactive effects on $\mathrm{P}_{\mathrm{n}}$. Incorporation of these models into the existing PhotoSim decision-support tool (USDA-ARS, 2019) is planned, to provide growers the opportunity to use these models. The interactive effect of light intensity, $\mathrm{CO}_{2}$ concentration, and leaf temperature indicates that growers should consider lighting, temperature control, and $\mathrm{CO}_{2}$ decisions jointly. If ambient $\mathrm{CO}_{2}$ concentrations in a greenhouse or controlled environment are sub-ambient, and they often are (Erwin and Gesick, 2017; Mortensen, 1987), the expected increase in $\mathrm{P}_{\mathrm{n}}$ with supplemental lighting will be tempered by limitations in $\mathrm{CO}_{2}$ fixation due to lack of available substrate. Similarly, low enzymatic activity at low ambient temperature can result in very low rates of $\mathrm{P}_{\mathrm{n}}$ despite high $\mathrm{CO}_{2}$ concentration and $P P F D$. Therefore, as sole-source or supplemental lighting guidelines are developed for crops, simultaneous consideration of $\mathrm{CO}_{2}$ concentration and temperature will be critical to achieve the ideal rates of $\mathrm{P}_{\mathrm{n}}$, either based on maximizing photosynthetic rate or optimizing the return on investment of inputs.

Future work measuring and modeling $\mathrm{P}_{\mathrm{n}}$ as a function of light intensity, $\mathrm{CO}_{2}$ concentration, and temperature would benefit from taking data points of these variables in combination, especially in relation to $\mathrm{CO}_{2}$ and temperature. Algorithm development and/or the use of surface plots could improve this type of modeling, as it would not require collection of data points at all possible combinations of variables and levels. In addition, including a sub-model to represent $\mathrm{R}_{\mathrm{d}}$ as a function of temperature and $\mathrm{CO}_{2}$ concentration might improve the fit of the Cannell and Thornley model.

\section{Literature Cited}

Abreu, P.P., M.M. Souza, A.A.F. de Almeida, E.A. Santos, J.C. de Oliveira, and A.L. Figueiredo. 2014. Photosynthetic responses of ornamental passion flower hybrids to varying light intensities. Acta Physiol. Plant. 36:1993-2004, https://doi.org/10.1007/s11738-014-1574-0.

Acock, B. 1991. Modeling canopy photosynthetic response to carbon dioxide, light interception, temperature and leaf traits, p. 41-55. In: K.J. Boote and R.S. Loomis (eds.). Modeling crop photosynthesis-From biochemistry to canopy. Crop Sci. Soc. Amer., Special Publ. No. 19. Amer. Soc. Agron., Madison, WI, https://doi.org/10.2135/ cssaspecpub19.c3.

Bezanson, J., S. Karpinski, V.B. Shah, and A. Edelman. 2012. Julia: A fast dynamic language for technical computing. 26 July 2021. $<$ https://arxiv.org/abs/1209.5145>.

Berry, J. and O. Björkman. 1980. Photosynthetic response and adaptation to temperature in higher plants. Annu. Rev. Plant Physiol. 31:491-543, https://doi.org/10.1146/annurev.pp.31.060180.002423.

Boldt, J.K., P.R. Fisher, and J.E. Erwin. 2014. Development of photosynthetic response curves and their integration into a decision- 
support tool for floriculture growers. HortScience 49(9):S389 (abstr.), https://doi.org/10.21273/HORTSCI.49.9S.S1.

Bunce, J.A. and G.H. Heichel. 1986. Measurements and modeling of photosynthesis in field crops. Crit. Rev. Plant Sci. 4:47-77, https:// doi.org/10.1080/07352688609382218.

Cannell, M.G.R. and J.H.M. Thornley. 1998. Temperature and $\mathrm{CO}_{2}$ responses of leaf and canopy photosynthesis: A clarification using the non-rectangular hyperbola model of photosynthesis. Ann. Bot. 82:883-892, https://doi.org/10.1006/anbo.1998.0777.

Delfine, S., F. Loreto, and A. Alvino. 2001. Drought-stress effects on physiology, growth and biomass production of rainfed and irrigated bell pepper plants in the Mediterranean region. J. Amer. Soc. Hort. Sci. 126:297-304, https://doi.org/10.21273/JASHS.126.3.297.

Dunning, I., J. Huchette, and M. Lubin. 2017. JuMP: A modeling language for mathematical optimization. Soc. Ind. Appl. Math. Rev. 59:295-320, https://doi.org/10.1137/15M1020575.

Erwin, J. and E. Gesick. 2017. Photosynthetic responses of swiss chard, kale, and spinach cultivars to irradiance and carbon dioxide concentration. HortScience 52:706-712, https://doi.org/10.21273/ HORTSCI11799-17.

Garcia, C. and R.G. Lopez. 2020. Supplemental radiation quality influences cucumber, tomato, and pepper transplant growth and development. HortScience 55:804-811, https://doi.org/10.21273/HORTSCI14820-20.

Idso, S.B., B.A. Kimball, M.G. Anderson, and J.R. Mauney. 1987. Effects of atmospheric $\mathrm{CO}_{2}$ enrichment on plant growth: The interactive role of air temperature. Agr. Ecosyst. Environ. 20:1-10, https:// doi.org/10.1016/0167-8809(87)90023-5.

Iwakiri, S. and M. Inayama. 1975. Studies on the canopy photosynthesis of the horticultural crops in controlled environment. J. Agr. Meteorol. 30:161-166, https://doi.org/10.2480/agrmet.30.161.

Johnson, I.R. and J.H.M. Thornley. 1984. A model of instantaneous and daily canopy photosynthesis. J. Theor. Biol. 107:531-545, https://doi. org/10.1016/S0022-5193(84)80131-9.

Johnson, I.R., J.H.M. Thornley, J.M. Frantz, and B. Bugbee. 2010. A model of canopy photosynthesis incorporating protein distribution through the canopy and its acclimation to light, temperature and $\mathrm{CO}_{2}$. Ann. Bot. 106:735-749, https://doi.org/10.1093/aob/mcq183.

Jurik, T.W., J.A. Weber, and D.M. Gates. 1988. Effects of temperature and light on photosynthesis of dominant species of a northern hardwood forest. Bot. Gaz. 149:203-208, https://doi.org/10.1086/337708.

Kimball, B.A. 1983. Carbon dioxide and agricultural yield: An assemblage and analysis of 430 prior observations. Agron. J. 75:779-788, https://doi.org/10.2134/agronj1983.00021962007500050014x.

Korczynski, P.C., J. Logan, and J.E. Faust. 2002. Mapping monthly distribution of daily light integrals across the contiguous United States. HortTechnology 12:12-16, https://doi.org/10.21273/HORTTECH.12.1.12.

Körner, O., E. Heuvelink, and Q. Niu. 2009. Quantification of temperature, $\mathrm{CO}_{2}$, and light effects on crop photosynthesis as a basis for model-based greenhouse climate control. J. Hort. Sci. Biotechnol. 84:233-239, https://doi.org/10.1080/14620316.2009.11512510.

McKinney, D. and J. Craver. 2021. The effect of $\mathrm{CO}_{2}$ enrichment timing and duration on pansy and petunia seedling quality for indoor production. HortScience 56(9):S267 (abstr.), https://doi.org/10.21273/ HORTSCI.56.9S.S1.

Mortensen, L.M. 1987. $\mathrm{CO}_{2}$ enrichment in greenhouses. Crop responses. Scientia Hort. 33:1-25, https://doi.org/10.1016/0304-4238(87)90028-8.

Owen, W.G. and H. Lindberg. 2017. Heat stress causes foliar bleaching and chlorosis of zonal geranium. e-GRO Alert 6(30):1-4. 26 July 2021. <http://www.e-gro.org/pdf/2017_630.pdf>.

Pachepsky, L.B. and B. Acock. 1996. An adequate model of photosynthesis - II. Dependence of parameters on environmental factors. Agr. Syst. 50:227-238, https://doi.org/10.1016/0308-521X(94)00052-S.

Peri, P.L., D.J. Moot, and D.L. McNeil. 2005. Modelling photosynthetic efficiency $(\alpha)$ for the light-response curve of cocksfoot leaves grown under temperate field conditions. Eur. J. Agron. 22:277-292, https://doi.org/10.1016/j.eja.2004.04.002.

Poorter, H., Ü. Niinemets, N. Ntagkas, A. Siebenkäs, M. Mäenpää, S. Matsubara, and T.L. Pons. 2019. A meta-analysis of plant responses to light intensity for 70 traits ranging from molecules to whole plant performance. New Phytol. 223:1073-1105, https://doi.org/10.1111/ nph. 15754.

Ronquim, C.C., C.H.B.A. Prado, and J. P.de Souza. 2009. Growth, photosynthesis and leaf water potential in young plants of Copaifera langsdorffii Desf. (Caesalpiniaceae) under contrasting irradiances. Braz. J. Plant Physiol. 21:197-208, https://doi.org/10.1590/ S1677-04202009000300004.

Seginer, I., G. Shina, L.D. Albright, and L.S. Marsh. 1991. Optimal temperature setpoints for greenhouse lettuce. J. Agr. Eng. Res. 49:209-226, https://doi.org/10.1016/0021-8634(91)80040-L.

Sinclair, T.R. and T. Horie. 1989. Leaf nitrogen, photosynthesis, and crop radiation use efficiency: A review. Crop Sci. 29:90-98, https:// doi.org/10.2135/cropsci1989.0011183X002900010023x.

Stanghellini, C. and J.A. Bunce. 1993. Response of photosynthesis and conductance to light, $\mathrm{CO}_{2}$, temperature and humidity in tomato plants acclimated to ambient and elevated $\mathrm{CO}_{2}$. Photosynthetica 29:487-497.

U.S. Department of Agriculture, Agricultural Research Service. 2019. PhotoSim. 26 July 2021. <https://data.nal.usda.gov/dataset/photosim >. von Caemmerer, S., G. Farquhar, and J. Berry. 2009. Biochemical model of $\mathrm{C}_{3}$ photosynthesis, p. 209-230. In: A. Laisk, L. Nedbal, and Govindjee (eds.). Photosynthesis in silico. Springer, Dordrecht, Netherlands, https://doi.org/10.1007/978-1-4020-9237-4_9.

Wächter, A. and L.T. Biegler. 2006. On the implementation of an interior-point filter line-search algorithm for large-scale nonlinear programming. Math. Program. 106:25-57, https://doi.org/10.1007/ s10107-004-0559-y.

Wullschleger, S.D. 1993. Biochemical limitations to carbon assimilation in $\mathrm{C}_{3}$ plants- $\mathrm{A}$ retrospective analysis of the $\mathrm{A} / \mathrm{C} i$ curves from 109 species. J. Expt. Bot. 44:907-920, https://doi.org/10.1093/jxb/44.5.907.

Yelle, S., R.C. Beeson, Jr., M.J. Trudel, and A. Gosselin. 1990. Duration of $\mathrm{CO}_{2}$ enrichment influences growth, yield, and gas exchange of two tomato species. J. Amer. Soc. Hort. Sci. 115:52-57, https:// doi.org/10.21273/JASHS.115.1.52. 